\title{
Nonlinear and Rate-Dependent Hysteretic Responses of Active Hybrid Composites
}

\author{
Chien-Hong Lin, Anastasia Muliana* \\ Department of Mechanical Engineering, Texas A\&M University, College Station, TX, USA \\ Email: *amuliana@neo.tamu.edu
}

Received 14 December 2015; accepted 26 January 2016; published 29 January 2016

Copyright (C 2016 by authors and Scientific Research Publishing Inc.

This work is licensed under the Creative Commons Attribution International License (CC BY). http://creativecommons.org/licenses/by/4.0/

(c) (i) Open Access

\begin{abstract}
Nonlinear electro-mechanical behaviors of piezoelectric materials and viscoelastic nature of polymers result in the overall nonlinear and hysteretic responses of active polymeric composites. This study presents a hybrid-unit-cell model for obtaining the effective nonlinear and rate-dependent hysteretic electro-mechanical responses of hybrid piezocomposites. The studied hybrid piezocomposites consist of unidirectional piezoelectric fibers embedded in a polymeric matrix, which is reinforced with piezoelectric particles. The hybrid-unit-cell model is derived based on a unit-cell model of fiber-reinforced composites consisting of fiber and matrix subcells, in which the matrix subcells are comprised of a unit-cell model of particle-reinforced composites. Nonlinear electro-mechanical responses are considered for the piezoelectric constituents while a viscoelastic solid constitutive model is used for the polymer constituent. The hybrid-unit cell model is used to examine the effects of different responses of the constituents, microstructural arrangements, and loading histories on the overall nonlinear and hysteretic electro-mechanical responses of the hybrid piezocomposites, which are useful in designing active polymeric composites.
\end{abstract}

\section{Keywords}

Piezoelectric, Polarization Switching, Micromechanics, Hybrid Composites, Nonlinear Electro-Mechanical Coupling, Viscoelasticity

\section{Introduction}

Piezoelectric fiber-reinforced composites have widely been used in aerospace, automobiles and medical industries due to their inherently large electro-mechanical coupling effects, compliant and lightweight characteristics. For example, a piezoelectric fiber-reinforced composite has a relatively high electro-mechanical coupling prop-

"Corresponding author. 
erty $d_{h} g_{h}{ }^{1}$, as reported by [1], and large in-plane actuations as experimentally observed by [2]. Polymers are often used as matrix in the piezoelectric fiber-reinforced composites. However, polymers have relatively low mechanical and electrical properties compared to those of piezoceramic fibers, such as lead zirconate titanate (PZT) fibers; thus, limiting the potential applications of the piezocomposites. Significant mismatches in the electro-mechanical properties of the fiber and matrix can lead to high stress discontinuities at the interface between the fibers and matrix, which can cause debonding. Neat polymeric matrix is often modified by adding particulate fillers in order to improve the properties of the polymeric matrix, which forms a hybrid composite with fibers and particles embedded in polymeric matrix. There have been several experimental studies on enhancing the mechanical properties of fibrous composites by dispersing particulate fillers into the matrix. For examples, hybrid composites show significant improvements in the transverse strength ([3] and [4]), the flexural strength [5]-[7], the longitudinal compressive strength [8]-[12], and the bearing strength [13]. References [14] and [2] have shown that improvement in the overall dielectric constants of a fibrous piezocomposite can be achieved by adding PZT powder with a dispersing agent into the epoxy matrix. Reference [2] also shows that a matrix system incorporating both dielectric and conductive fillers reduces the magnitude of voltages required for poling the fibrous piezocomposites. Reference [15] discusses that fibrous piezocomposites, which have relatively low transverse stiffness, are unable to bear large transverse loads without any additional substrates to enhance the structural stiffness.

Micromechanical models have been used to determine the overall electro-mechanical properties of piezocomposites, which focus mainly on the linear electro-mechanical responses of two-phase piezocomposites, e.g., [14] [16]-[19], and [21]-[23] for example. Nonlinear electro-mechanical responses of the two-phase piezocomposites with elastic or viscoelastic polymeric matrices are also studied using micromechanical models, which can be found in [23]-[26]. There have been limited micromechanical models for predicting the overall hysteretic polarization response, i.e., polarization switching behavior, of unidirectional piezoelectric fiber composites, e.g., [19]-[27]. The above studies consider rate-independent electro-mechanical response of the piezoelectric constituent. While extensive micromechanics studies have been done on understanding responses of piezoelectric fiber composites, only limited micromechanical models are available for predicting the overall responses of hybrid piezocomposites, i.e., active composites comprising of multiple types and shapes of inclusions/inhomogeneities. Reference [30] uses the correspondence principle in conjunction with the Mori-Tanaka model to evaluate the effective loss factor of a hybrid piezocomposite having shunted piezoelectric particles embedded in a conductive particle reinforced matrix. This model is extended by [31] to derive the effective loss factor for a hybrid piezocomposite with orientation-dependent piezoelectric inhomogeneities and conductive inhomogeneities dispersed in a viscoelastic polymer.

In many applications, hybrid piezocomposites consisting of PZT inhomogeneities and polymeric matrix are often exposed to various mechanical and electrical stimuli. Large electric driving fields can cause significant nonlinear strain responses of polarized PZTs [32], which are often a case in actuators. A polarized PZT may be depolarized if an electric field that is greater than the coercive field limit of the material is applied opposite to the current poling direction, or if a relatively high compressive load is applied along the poling axis, or if its operating temperature exceeds the Curie temperature. Depending on the magnitude and duration of exposure to the external stimuli and boundary conditions, PZTs can exhibit time-dependent and nonlinear electro-mechanical coupling effects, and the polymeric matrix can experience pronounced viscoelastic behavior. Therefore, hybrid piezocomposites can experience overall nonlinear time-dependent and hysteretic electro-mechanical responses. It is then necessary to study the overall nonlinear and rate-dependent hysteretic behaviors of the hybrid piezocomposites prior to designing and fabricating smart devices made of these piezocomposites, which is currently limited.

This study presents formulations of a hybrid unit-cell model for determining the effective nonlinear and hysteretic responses of hybrid piezocomposites, which consist of unidirectional piezoelectric fibers embedded in a viscoelastic polymeric matrix reinforced with piezoelectric particle fillers, subjected to high electric fields and mechanical stresses. In this paper, fibers and particles are made of PZTs; however, the unit-cell model formulation is general and can incorporate different piezoelectric materials for the different inhomogeneities. We consider both nonlinear electro-mechanical response of polarized PZTs and polarization switching behavior of PZTs under large cyclic electric field inputs. This article is organized as follows: Section 2 briefly discusses the con-

\footnotetext{
${ }^{1}$ This parameter is used for measuring the efficiency of a piezoceramic for ultrasonic applications and for hydrophone devices in particular.
} 
stitutive models for the constituents followed by numerical methods for solving the coupled nonlinear electromechanical constitutive models in Section 3. Section 4 presents the formulation of the hybrid-unit-cell model for obtaining the effective nonlinear and rate-dependent hysteretic response of composites. Numerical results on the effective responses of the hybrid piezocomposites are discussed in Section 5. Section 6 is dedicated to conclusions.

\section{Constitutive Models for the Constituents}

PZTs are polarized by applying high electric field, above the coercive electric field at elevated temperature [33] before they are used in sensing and actuating applications. The polarized PZTs show electro-mechanical coupling response, which is quantified by piezoelectric constants. When high electric field is prescribed to the polarized PZTs, which is often the case in actuator applications, they exhibit nonlinear electro-mechanical coupling response. In this study, we use the constitutive model proposed by [34] for modeling nonlinear responses of polarized PZTs subjected to large electric field but smaller than coercive electric field of the PZTs, which is within a range in practical applications. Another type of nonlinear electro-mechanical coupling response is hysteretic polarization switching response. Polarization switching can occur when high amplitude of cyclic electric field above the coercive electric field of the materials is considered. We adopt the constitutive model proposed by [35] for modeling the hysteretic polarization switching of PZTs. Finally a linear viscoelastic constitutive model is used for the polymer constituent.

\subsection{Polarized PZTs}

A nonlinear constitutive model proposed by [34] for polarized PZTs undergoing large electric fields and small strains is given as:

$$
\begin{gathered}
\varepsilon_{i j}=s_{i j k l} \sigma_{k l}+d_{k i j} E_{k}+\frac{1}{2} f_{k l i j} E_{l} E_{k}, \\
D_{i}=d_{i k l} \sigma_{k l}+\kappa_{i j} E_{j}+\frac{1}{2} \chi_{i j k} E_{k} E_{j},
\end{gathered}
$$

where $\varepsilon_{i j}, \sigma_{i j}, E_{i}$ and $D_{i}$ are the scalar components of strain, stress, electric field and electric displacement, respectively. The material properties are the elastic compliances $s_{i j k l}$ determined at a constant electric field, the third- and fourth-order piezoelectric strain coefficients $d_{i j k}$ and $f_{i j k l}$, respectively, which are determined at constant stresses; and the second- and third-order dielectric coefficients $\kappa_{i j}$ and $\chi_{i j k}$ calibrated at constant stresses. The higher-order term of the electric field is introduced in order to better capture the nonlinear response of the polarized PZTs due to large electric driving fields.

\subsection{Hysteretic Polarization Switching of PZTs}

A rate-dependent electro-mechanical constitutive model, incorporating polarization switching response, formulated by [35], is given as:

$$
\begin{gathered}
\varepsilon_{i j}^{t} \equiv \varepsilon_{i j}(t)=s_{i j k l} \sigma_{k l}^{t}+4 g_{n i j}^{t} \kappa_{n m} g_{m k l}^{t} \sigma_{k l}^{t}+g_{k i j}^{t} P_{k}^{t}, \\
D_{i}^{t}=2 \kappa_{i m} g_{m k l}^{t} \sigma_{k l}^{t}+P_{i}^{t},
\end{gathered}
$$

where $g_{i j k}^{t}$ is the scalar component of the third-order piezoelectric coefficient which is dependent on the current polarization $P_{3}^{t}$ with the $x_{3}$ direction chosen as the poling axis. The upper right superscript $t$ indicates the current time. The piezoelectric constant $g_{i j k}^{t}$ is assumed as:

$$
g_{i j k}^{t}=\frac{P_{3}^{t}}{P_{r}} \mathrm{e}^{\frac{-\left|P_{3}^{t}\right|}{c_{1}}} g_{i j k}^{r},
$$

where $g_{i j k}^{r}$ is the scalar component of the third-order piezoelectric coefficient measured at constant (remanent) polarization $P_{r}$. It is noted that $\mathbf{g}^{r}=\boldsymbol{\kappa}^{-1} \mathbf{d}^{r}$, where $\mathbf{d}^{r}$ is the direct piezoelectric constant measured at remanent polarization. The scalar components of the polarization are 


$$
\begin{gathered}
P_{1}^{t}=\kappa_{11} E_{1}^{t}, \\
P_{2}^{t}=\kappa_{22} E_{2}^{t} . \\
P_{3}^{t}=R\left(E_{3}^{\tau}, t\right)+Q\left(E_{3}^{t}\right),
\end{gathered}
$$

where $R\left(E_{3}^{\tau}, t\right)$ is the time-dependent reversible polarization at current time $t$ with $R(0, t)=0$ while $Q\left(E_{3}^{t}\right)$ is the residual (irreversible) polarization. The upper right superscript $\tau$ denotes the previous time variable. The reversible polarization is written as:

$$
R^{t}=R\left(E_{3}^{0}, t\right)+\int_{0}^{t} \frac{\partial}{\partial E_{3}^{\tau}} R\left(E_{3}^{\tau}, t-\tau\right) \frac{\mathrm{d} E_{3}^{\tau}}{\mathrm{d} \tau} \mathrm{d} \tau,
$$

where

$$
R\left(E_{3}^{0}, t\right)=R_{0}\left(E_{3}^{0}\right)+R_{1}\left(E_{3}^{0}\right)\left(1-\exp \left(-\frac{t}{\tau_{1}}\right)\right) .
$$

Both $R_{0}\left(E_{3}^{\tau}\right)$ and $R_{1}\left(E_{3}^{\tau}\right)$ are function of $E_{3}^{\tau}$. The characteristic time $\tau_{1}$ indicates the speed of polarization changes. The irreversible polarization is given as:

$$
Q^{t}=\int_{0}^{E_{3}^{t}} \frac{\mathrm{d} Q^{\tau}}{\mathrm{d} E_{3}^{\tau}} \mathrm{d} E_{3}^{\tau} .
$$

The rate of the residual polarization during polarization switching response is:

$$
\frac{\mathrm{d} Q^{t}}{\mathrm{~d} E_{3}^{t}}= \begin{cases}0, & 0 \leq E_{3}^{t}<E_{m}, \mathrm{~d} E_{3}^{t}<0 \text { or }-E_{m}<E_{3}^{t} \leq 0, \mathrm{~d} E_{3}^{t}>0, \\ \lambda \mid \frac{\left.E_{3}^{t}\right|^{n}}{\left.E_{c}\right|^{n},} & -E_{c} \leq E_{3}^{t}<0, \mathrm{~d} E_{3}^{t} \leq 0 \text { or } 0<E_{3}^{t} \leq E_{c}, \mathrm{~d} E_{3}^{t} \geq 0, \\ \mu \exp \left[-\omega\left(\frac{\left|E_{3}^{t}\right|}{E_{c}}-1\right)\right], & -E_{m} \leq E_{3}^{t}<-E_{c}, \mathrm{~d} E_{3}^{t} \leq 0 \text { or } E_{c}<E_{3}^{t} \leq E_{m}, \mathrm{~d} E_{3}^{t} \geq 0,\end{cases}
$$

where $\lambda, \mu, \omega, n$ are the material parameters that are calibrated from experiments. A similar function with different material parameters can be used for modeling the initial polarization, as discussed in [36].

The compressive stresses along the poling axis could significantly affect the hysteretic polarization switching response. In this study, it is assumed that the coercive electric field varies with the compressive stresses along the $x_{3}$ direction:

$$
E_{c}= \begin{cases}E_{c}\left(E_{c}^{0}, \sigma_{33}^{t}\right), & \sigma_{33}^{t}<0, \\ E_{c}^{0}, & \sigma_{33}^{t} \geq 0,\end{cases}
$$

where $E_{c}^{0}$ is the coercive electric field in absence of mechanical stresses. In order to incorporate the effect of compressive stress on the polarization switching responses, it is assumed that the compressive stress that is higher than the coercive stress limit affects the current polarization state $P_{3}^{t}$ and the piezoelectric coefficient $g_{i j k}^{t}$ :

$$
g_{i j k}^{t}=\frac{P_{3}^{t}}{P_{r}} \mathrm{e}^{\frac{-\left|P_{3}^{t}\right|}{c_{1}}} \mathrm{e}^{\frac{-c_{2}\left|\sigma_{33}^{t}\right|}{\sigma_{c}}} g_{i j k}^{r}, \quad c_{2}=0, \quad \text { if } \sigma_{33}^{t}>-\sigma_{c},
$$

where $\sigma_{c}$ is the coercive stress limit and $C_{2}$ is a material parameter. Figure 1 shows that the rate-dependent electro-mechanical constitutive model can capture the hysteretic polarization and butterfly strain responses of a stress free PZT-51 undergoing a cyclic electric field input. The experimental data of the PZT-51 are obtained from [37]. The material parameters used to capture the hysteretic polarization and strain responses are given in Tables 1-4. 

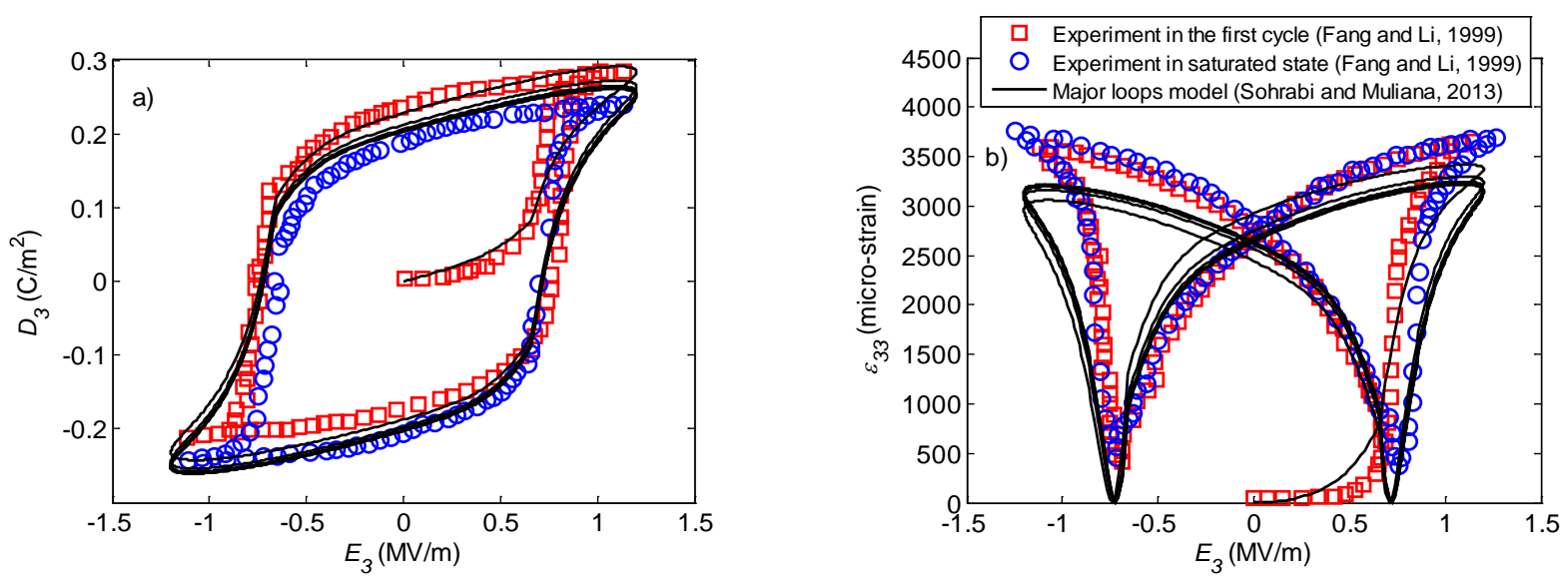

Figure 1. (a) Hysteretic polarization and (b) butterfly strain responses for a stress free PZT-51.

Table 1. Material parameters for the time-dependent polarization of PZT-51 ([35]).

\begin{tabular}{cccccccc}
\hline $\begin{array}{c}E_{c}^{0} \\
(\mathrm{MV} / \mathrm{m})\end{array}$ & $\begin{array}{c}\kappa_{0} \\
\left(\times 10^{-9} \mathrm{~F} / \mathrm{m}\right)\end{array}$ & $\begin{array}{c}\kappa_{1} \\
\left(\times 10^{-9} \mathrm{~F} / \mathrm{m}\right)\end{array}$ & $\begin{array}{c}\tau_{1} \\
(\mathrm{sec})\end{array}$ & $\begin{array}{c}\lambda \\
\left(\times 10^{-6} \mathrm{~F} / \mathrm{m}\right)\end{array}$ & $n$ & $\begin{array}{c}\mu \\
\left(\times 10^{-6} \mathrm{~F} / \mathrm{m}\right)\end{array}$ & $\omega$ \\
\hline 0.67 & 70 & 225 & 1 & 0.35 & 3 & 1.6 & 4 \\
\hline
\end{tabular}

Table 2. Electro-mechanical coupling parameters for PZT-51 ([35]).

\begin{tabular}{cccccc}
\hline $\begin{array}{c}d_{333}^{r} \\
\left(\times 10^{-12} \mathrm{~m} / \mathrm{V}\right)\end{array}$ & $\begin{array}{c}d_{311}^{r} \\
\left(\times 10^{-12} \mathrm{~m} / \mathrm{V}\right)\end{array}$ & $\begin{array}{c}\kappa_{11}^{r} \\
\left(\times 10^{-9} \mathrm{~F} / \mathrm{m}\right)\end{array}$ & $\begin{array}{c}\kappa_{33}^{r} \\
\left(\times 10^{-9} \mathrm{~F} / \mathrm{m}\right)\end{array}$ & $\begin{array}{c}P_{r} \\
\left(\mathrm{C} / \mathrm{m}^{2}\right)\end{array}$ & $C_{1}$ \\
\hline 1520 & -570 & 38 & 42 & 0.194 & 0.19 \\
\hline
\end{tabular}

Table 3. Elastic constants for PZT-51 ([35]).

\begin{tabular}{cccccc}
\hline $\begin{array}{c}E_{11}=E_{22} \\
(\mathrm{GPa})\end{array}$ & $\begin{array}{c}E_{33} \\
(\mathrm{GPa})\end{array}$ & $\begin{array}{c}G_{12} \\
(\mathrm{GPa})\end{array}$ & $\begin{array}{c}G_{31}=G_{32} \\
(\mathrm{GPa})\end{array}$ & $v_{12}$ & $v_{31}=v_{32}$ \\
\hline 34.48 & 33.00 & 13.19 & 12.37 & 0.307 & 0.334 \\
\hline
\end{tabular}

Table 4. Material parameters above the coercive stress limit for PZT-51 ([35]).

\begin{tabular}{cccccc}
\hline $\begin{array}{c}\sigma_{c} \\
(\mathrm{MPa})\end{array}$ & $C_{2}$ & $\begin{array}{c}\lambda \\
\left(\times 10^{-6} \mathrm{~F} / \mathrm{m}\right)\end{array}$ & $n$ & $\mu$ & $\omega$ \\
\hline 25 & 0.3 & 0.40 & 3 & 1.1 & 4 \\
\hline
\end{tabular}

\subsection{Polymers}

The polymeric matrix is assumed as an isotropic viscoelastic solid, which is:

$$
\begin{gathered}
\varepsilon_{i j}^{t}=\int_{0}^{t} \frac{1}{2} J(t-\tau) \frac{\mathrm{d} S_{i j}^{\tau}}{\mathrm{d} \tau} \mathrm{d} \tau+\frac{1}{3} \delta_{i j} \int_{0}^{t} \frac{1}{3} B(t-\tau) \frac{\mathrm{d} \sigma_{k k}^{\tau}}{\mathrm{d} \tau} \mathrm{d} \tau, \\
D_{i}=\kappa_{i j} E_{j},
\end{gathered}
$$

where $\delta_{i j}$ is the Kronecker delta. $J(t)$ and $B(t)$ are the time-dependent shear and bulk compliances, respectively. $S_{i j}^{t}$ and $\sigma_{k k}^{t}$ are the scalar components of the deviatoric and volumetric stress tensors at time $t$, respectively. To reduce complexity in modeling the viscoelastic response of the hybrid piezocomposites, we shall assume that the corresponding linear elastic Poisson's ratio $v$ for the polymers is time-independent. The 
shear and bulk compliance share the same time function as the extensional (uniaxial) compliance:

$$
\begin{aligned}
& J(t)=2(1+v) D(t), \\
& B(t)=3(1-2 v) D(t),
\end{aligned}
$$

where $D(t)$ is the time-dependent uniaxial compliance, which is expressed as:

$$
D(t)=D_{0}+\sum_{n=1}^{N} D_{n}\left(1-\exp \left[-\lambda_{n} t\right]\right)
$$

Here $D_{0}$ is the instantaneous (elastic) compliance and the transient compliance is expressed in terms of a series of exponential functions, where $N$ is the number of terms, $D_{n}$ is the $n^{\text {th }}$ coefficient of the time-dependent compliance and $\lambda_{n}$ is the $n^{\text {th }}$ reciprocal of retardation time.

\section{Linearized Forms of the Nonlinear Constitutive Models}

For convenience in analyzing the time-dependent and nonlinear electro-mechanical behavior, we present a linearized incremental form of the constitutive relations, i.e., Equations (1), (2), (3), (4), (15) and (16). A recursive time-integration algorithm presented in [38] is used to numerically evaluate the time integral forms of the constitutive models such as for Equations (9) and (15). The incremental independent field variables at current time $t$ are:

$$
\begin{aligned}
\Delta \boldsymbol{\sigma}^{t} & =\boldsymbol{\sigma}^{t}-\boldsymbol{\sigma}^{t-\Delta t}, \\
\Delta \mathbf{E}^{t} & =\mathbf{E}^{t}-\mathbf{E}^{t-\Delta t},
\end{aligned}
$$

where superscript $t-\Delta t$ denotes the previous time and $\Delta t$ is the current incremental time.

The linearized constitutive relation can be expressed in a single equation, which follows a conventional indicial notation with lower case subscripts range from 1 to 3 while upper case subscripts range from 1 to 4 :

$$
\Xi_{i J}^{t}=O_{i J M n}^{t} \Delta T_{M n}^{t}+\Xi_{i J}^{t-\Delta t},
$$

where

$$
\begin{gathered}
\Xi_{i J}^{t}= \begin{cases}\varepsilon_{i j}^{t}, & J=1,2,3, \\
D_{i}^{t}, & J=4,\end{cases} \\
O_{i J M n}^{t}= \begin{cases}\tilde{s}_{i j n n}^{t}, & J, M=1,2,3, \\
\tilde{d}_{n i j}^{\prime t}, & J=1,2,3 ; M=4, \\
\tilde{d}_{i m n}^{t}, & J=4 ; M=1,2,3, \\
\tilde{\kappa}_{i n}^{t}, & J, M=4,\end{cases} \\
\Delta T_{M n}^{t}= \begin{cases}\Delta \sigma_{m n}^{t}, & M=1,2,3, \\
\Delta E_{n}^{t}, & M=4 .\end{cases}
\end{gathered}
$$

The components of $O_{i J M n}^{t}$ are represented by a 9 by 9 matrix. Vectors $\Xi_{i J}^{t}$ and $\Delta T_{M n}^{t}$ are 9 by 1 column vectors and $\Xi_{i J}^{t-\Delta t}$ is the history variables of the dependent field variables $\Xi_{i J}^{t}$. A factor of two for the shear strains is accounted for in the vector $\Xi_{i J}^{t}$. This matrix formulation of the linearized constitutive relation will be used in the following micromechanical analysis. After some algebraic manipulations, the resulting components of $O_{i J M n}^{t}$ and $\Xi_{i J}^{t-\Delta t}$ for each constitutive model are summarized as:

\subsection{Polarized PZTs}

From Equations (1) and (2), the resulting components of $O_{i J M n}^{t}$ and $\Xi_{i J}^{t-\Delta t}$ are: 


$$
\begin{aligned}
& \tilde{s}_{i j m n}^{t}=s_{i j m n}, \\
& \tilde{d}_{n i j}^{\prime t}=d_{n i j}+\frac{1}{2} f_{n l i j} E_{l}^{t}, \\
& \tilde{d}_{i m n}^{t}=d_{i m n}, \\
& \tilde{\kappa}_{i n}^{t}=\kappa_{i n}+\frac{1}{2} \chi_{i n k} E_{k}^{t}, \\
& \varepsilon_{i j}^{t-\Delta t}=s_{i j k l} \sigma_{k l}^{t-\Delta t}+\left(d_{k i j}+\frac{1}{2} f_{k l i j} E_{l}^{t}\right) E_{k}^{t-\Delta t}, \\
& D_{i}^{t-\Delta t}=d_{i k l} \sigma_{k l}^{t-\Delta t}+\left(\kappa_{i j}+\frac{1}{2} \chi_{i j k} E_{k}^{t}\right) E_{j}^{t-\Delta t} .
\end{aligned}
$$

\subsection{Hysteretic Polarization Switching Response}

From Equations (3) and (4), the resulting components of $O_{i J M n}^{t}$ and $\Xi_{i J}^{t-\Delta t}$ are:

$$
\begin{aligned}
& \tilde{s}_{i j m n}^{t}=s_{i j m n}+4 g_{k i j}^{t} \kappa_{k l} g_{l m n}^{t}, \\
& \tilde{d}_{n i j}^{t t}=g_{n i j}^{t} K_{k l}^{t}, \\
& \tilde{d}_{i m n}^{t}=2 \kappa_{i j} g_{j m n}^{t}, \\
& \tilde{\kappa}_{i n}^{t}=K_{i n}^{t}, \\
& \varepsilon_{i j}^{t-\Delta t}=\left(s_{i j k l}+4 g_{n i j}^{t} \kappa_{n m} g_{m k l}^{t}\right) \sigma_{k l}^{t-\Delta t}+g_{k i j}^{t}\left(P_{k}^{t-\Delta t}+F_{k}^{t}\right), \\
& D_{i}^{t-\Delta t}=2 \kappa_{i m} g_{m k l}^{t} \sigma_{k l}^{t-\Delta t}+P_{i}^{t-\Delta t}+F_{i}^{t},
\end{aligned}
$$

where $R_{0}\left(E_{3}^{\tau}\right)$ and $R_{1}\left(E_{3}^{\tau}\right)$ in Equation (10) are considered as linear functions:

$$
\begin{aligned}
& R_{0}\left(E_{3}^{\tau}\right)=\kappa_{0} E_{3}^{\tau}, \\
& R_{1}\left(E_{3}^{\tau}\right)=\kappa_{1} E_{3}^{\tau},
\end{aligned}
$$

where $\kappa_{0}$ is the dielectric constant of a macroscopically unpolarized PZT and $\kappa_{1}$ is the time-dependent part of the dielectric constant. In Equation (27) $K_{i j}^{t}$ is:

$$
K_{i j}^{t}= \begin{cases}\kappa_{11}, & i=j=1, \\ \kappa_{22}, & i=j=2, \\ \kappa_{0}+\kappa_{1}+\Delta Q^{t}, & i=j=3, \\ 0, & i \neq j .\end{cases}
$$

Using the rate of residual polarization in Equation (12), the incremental residual polarization at current time $t$ is approximated by:

$$
\Delta Q^{t} \approx \frac{\mathrm{d} Q^{t}}{\mathrm{~d} E_{3}^{t}} \Delta E_{3}^{t}
$$

Finally, $F_{i j}^{t}$ and $P_{i}^{t}$ are expressed as:

$$
F_{i}^{t}=\left\{\begin{array}{cc}
0, & i=1,2, \\
{\left[1-\exp \left[-\frac{\Delta t}{\tau_{1}}\right]\right] q^{t-\Delta t}-\left[\frac{\partial R_{1}\left(E_{3}^{t}\right)}{\partial E_{3}^{t}} \frac{\mathrm{d} E_{3}^{t}}{\mathrm{~d} t}+\exp \left[-\frac{\Delta t}{\tau_{1}}\right] \frac{\partial R_{1}\left(E_{3}^{t-\Delta t}\right)}{\partial E_{3}^{t-\Delta t}} \frac{\mathrm{d} E_{3}^{t-\Delta t}}{\mathrm{~d} t}\right] \frac{\Delta t}{2}, \quad i=3,} & P_{i}^{t}=P_{i}^{t-\Delta t}+\Delta P_{i}^{t},
\end{array}\right.
$$

where the history variable related to the polarization is: 


$$
q^{t}=\exp \left[-\frac{\Delta t}{\tau_{1}}\right] q^{t-\Delta t}+\left[\frac{\partial R_{1}\left(E_{3}^{t}\right)}{\partial E_{3}^{t}} \frac{\mathrm{d} E_{3}^{t}}{\mathrm{~d} t}+\exp \left[-\frac{\Delta t}{\tau_{1}}\right] \frac{\partial R_{1}\left(E_{3}^{t-\Delta t}\right)}{\partial E_{3}^{t-\Delta t}} \frac{\mathrm{d} E_{3}^{t-\Delta t}}{\mathrm{~d} t}\right] \frac{\Delta t}{2},
$$

and the incremental polarization is determined by:

$$
\Delta P_{i}^{t}=K_{i j}^{t} \Delta E_{j}^{t}+F_{i}^{t}
$$

\subsection{Polymers}

From Equations (15) and (16), the resulting components of $O_{i J M n}^{t}$ and $\Xi_{i J}^{t-\Delta t}$ are:

$$
\begin{aligned}
& \tilde{s}_{i j m n}^{t}=\left(\frac{1}{3} \breve{B}-\frac{1}{3} \breve{J}\right) \delta_{i j} \delta_{m n}+2 \breve{J} \delta_{i m} \delta_{j n}-\breve{J} \delta_{i n} \delta_{j m}, \\
& \tilde{d}_{n i j}^{t t}=0, \\
& \tilde{d}_{i m n}^{t}=0, \\
& \tilde{\kappa}_{i n}^{t}=\kappa_{i n}^{t}, \\
& \varepsilon_{i j}^{t-\Delta t}=2\left(\breve{J} S_{i j}^{t-\Delta t}-d_{i j}^{t}\right)+\delta_{i j}\left[-\breve{J} S_{i j}^{t-\Delta t}+d_{i j}^{t}+\frac{1}{3}\left(\breve{B} \sigma_{k k}^{t-\Delta t}-V_{k k}^{t}\right)\right], \\
& D_{i}^{t-\Delta t}=\kappa_{i j} E_{j}^{t-\Delta t} .
\end{aligned}
$$

$\breve{B}, \breve{J}, d_{i j}^{t}$ and $V_{k k}^{t}$ in Equation (35) are given as:

$$
\begin{aligned}
& \breve{B}^{t}=(1-2 v)\left[D_{0}+\sum_{n=1}^{N} D_{n}-\sum_{n=1}^{N} D_{n} \frac{1-\exp \left[-\lambda_{n} \Delta t\right]}{\lambda_{n} \Delta t}\right], \\
& \breve{J}^{t}=(1+v)\left[D_{0}+\sum_{n=1}^{N} D_{n}-\sum_{n=1}^{N} D_{n} \frac{1-\exp \left[-\lambda_{n} \Delta t\right]}{\lambda_{n} \Delta t}\right], \\
& d_{i j}^{t}=(1+v) \sum_{n=1}^{N} J_{n}\left[\exp \left[-\lambda_{n} \Delta t\right] q_{i j, n}^{t-\Delta t}-\frac{1-\exp \left[-\lambda_{n} \Delta t\right]}{\lambda_{n} \Delta t} S_{i j}^{t-\Delta t}\right], \\
& V_{k k}^{t}=(1-2 v) \sum_{n=1}^{N} J_{n}\left[\exp \left[-\lambda_{n} \Delta t\right] q_{k k, n}^{t-\Delta t}-\frac{1-\exp \left[-\lambda_{n} \Delta t\right]}{\lambda_{n} \Delta t} \sigma_{k k}^{t-\Delta t}\right],
\end{aligned}
$$

where the history variables related to the deviatoric and volumetric strains are:

$$
\begin{aligned}
& q_{i j, n}^{t}=\exp \left[-\lambda_{n} \Delta t\right] q_{i j, n}^{t-\Delta t}+\left[\frac{1-\exp \left[-\lambda_{n} \Delta t\right]}{\lambda_{n} \Delta t}\right]\left(S_{i j}^{t}-S_{i j}^{t-\Delta t}\right), \\
& q_{k k, n}^{t}=\exp \left[-\lambda_{n} \Delta t\right] q_{k k, n}^{t-\Delta t}+\left[\frac{1-\exp \left[-\lambda_{n} \Delta t\right]}{\lambda_{n} \Delta t}\right]\left(\sigma_{k k}^{t}-\sigma_{k k}^{t-\Delta t}\right) .
\end{aligned}
$$

\section{Hybrid-Unit-Cell Model}

This section presents formulations of a hybrid-unit-cell model for obtaining the overall responses of hybrid piezocomposites whose constituents experience nonlinear electro-mechanical and viscoelastic behaviors. The microstructures of a hybrid piezocomposite are idealized with periodically distributed fibers of square cross section in a matrix medium and the microstructures of the matrix are idealized with periodically distributed cubic particles in a homogeneous viscoelastic matrix. Here, we consider a unit cell as the smallest representative microstructures and each unit cell is divided into several subcells. Figure 2 illustrates an idealized unit-cell model of the hybrid piezocomposites. At the upper scale, a hybrid-unit-cell model consists of a fiber unit-cell, comprising of four fiber and matrix subcells, and the lower scale is a particle-unit-cell model, having eight particle and polymer subcells. The particle unit-cell model is implemented at each matrix subcell in the fiber unit-cell model. 


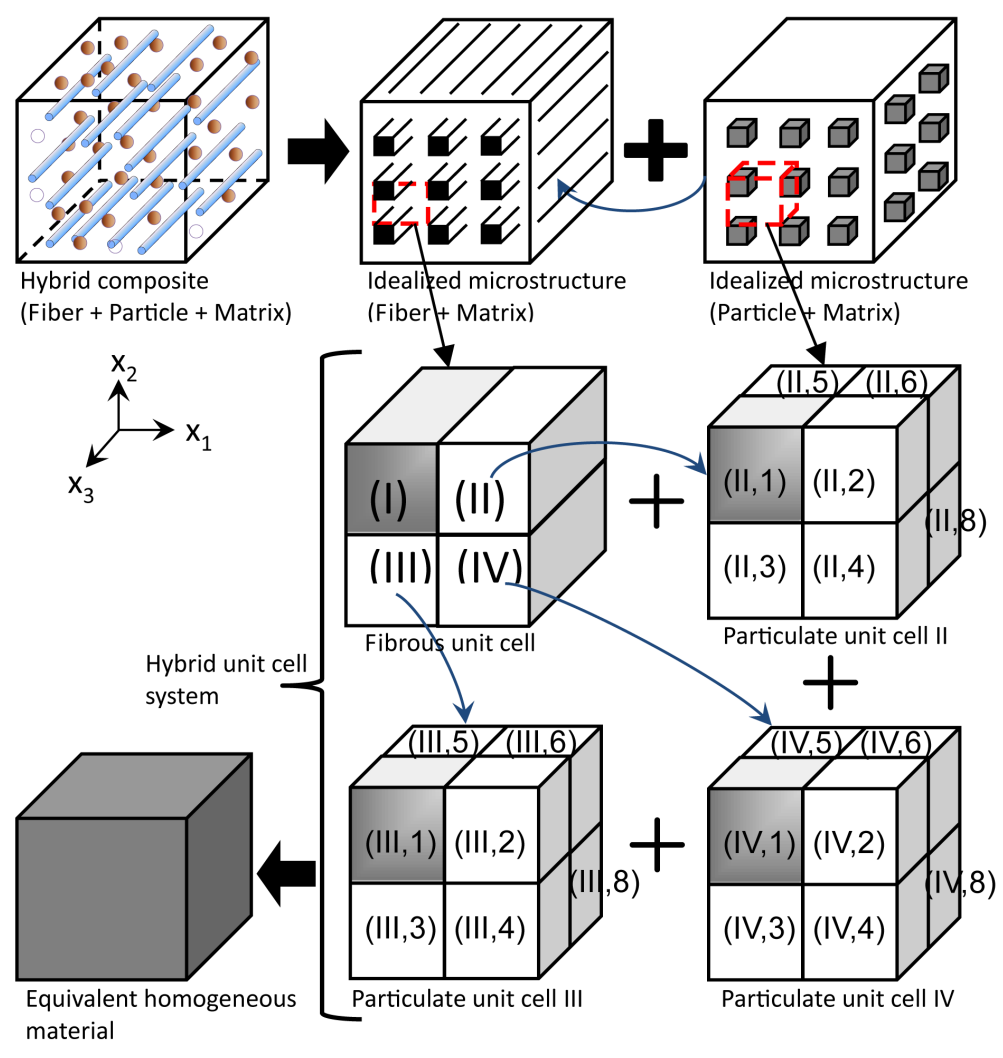

Figure 2. Hybrid-unit-cell model.

The first subcell of the fiber unit cell is the piezoelectric fiber constituent and the rest of the subcells represent the matrix, whose response is determined from a homogenized active composite of the particulate unit cells. The first subcell of the particulate unit cell is the piezoelectric particle constituent and the remaining subcells in the particulate unit cell indicate the homogeneous viscoelastic matrix. The fibrous and particulate unit cells lead to rather simple micromechanical relations by satisfying equilibrium condition and displacement compatibility among all subcells. The time-integration algorithm for the rate-dependent PZT (Equation (9)) and viscoelastic matrix (Equation (15)) is nested to the hybrid-unit-cell model in order to obtain approximate solutions of the overall nonlinear and time-dependent responses of the hybrid piezocomposites. The cross section of the fiber is assumed to be square and the one of the particle is taken to be a cube, which is done to simplify the micromechanics formulation. In the previous work by one of the author ([27]) shows that this geometrical simplification gives very good predictions of the overall polarization switching responses of active fiber composites when compared to experimental data. This means that for mainly determining the overall nonlinear responses of composites the effect of detailed shapes of the cross-sectional geometries of the inclusions is rather insignificant.

For the derivation of the hybrid-unit-cell model, we start with the fibrous unit cell. Using a volume-average scheme, the effective field variable, denoted by an overbar, of the fibrous unit cell at current time $t$ is written as:

$$
\overline{\boldsymbol{\Xi}}^{t}=\sum_{\alpha=1}^{\mathrm{IV}} c^{(\alpha)} \boldsymbol{\Xi}^{(\alpha), t}
$$

The superscript $(\alpha)$ denotes the subcell's number of the fibrous unit cell. The fiber volume fraction is defined as $c^{(\mathrm{I})}=V^{(\mathrm{I})} / V$ (i.e., volume fraction of the fibers with respect to the hybrid piezocomposite) and the fibrous unit cell volume is given by $V=\sum_{\alpha=1}^{\alpha=I V} V^{(\alpha)}$. A linearized constitutive relation for the fibrous piezocomposite at current time $t$ is written as:

$$
\overline{\mathbf{\Xi}}^{t}=\overline{\mathbf{O}}^{t} \Delta \overline{\mathbf{T}}^{t}+\overline{\mathbf{\Xi}}^{t-\Delta t},
$$

and also for the subcell $(\alpha)$ is: 


$$
\boldsymbol{\Xi}^{(\alpha), t}=\mathbf{O}^{(\alpha), t} \Delta \mathbf{T}^{(\alpha), t}+\mathbf{\Xi}^{(\alpha), t-\Delta t} .
$$

In order to relate the effective incremental independent field variables in the fibrous unit cell to the corresponding incremental field variables in its subcells, a concentration matrix $\mathbf{B}^{(\alpha), t}$ and a vector of history variable $\mathbf{X}^{(\alpha), t}$ at current time $t$ are defined through the relation:

$$
\Delta \mathbf{T}^{(\alpha), t}=\mathbf{B}^{(\alpha), t} \Delta \overline{\mathbf{T}}^{t}+\mathbf{X}^{(\alpha), t} .
$$

Substituting $\Delta \mathbf{T}^{(\alpha), t}$ from Equation (41) into (40) gives

$$
\boldsymbol{\Xi}^{(\alpha), t}=\mathbf{O}^{(\alpha), t} \mathbf{B}^{(\alpha), t} \Delta \overline{\mathbf{T}}^{t}+\mathbf{O}^{(\alpha), t} \mathbf{X}^{(\alpha), t}+\mathbf{\Xi}^{(\alpha), t-\Delta t} .
$$

Substituting $\boldsymbol{\Xi}^{(\alpha), t}$ from Equation (42) into (38) gives

$$
\bar{\Xi}^{t}=\sum_{\alpha=\mathrm{I}}^{\mathrm{IV}} c^{(\alpha)} \mathbf{O}^{(\alpha), t} \mathbf{B}^{(\alpha), t} \Delta \overline{\mathbf{T}}^{t}+\sum_{\alpha=\mathrm{I}}^{\mathrm{IV}} c^{(\alpha)}\left(\mathbf{O}^{(\alpha), t} \mathbf{X}^{(\alpha), t}+\mathbf{\Xi}^{(\alpha), t-\Delta t}\right) .
$$

From Equations (43) and (39), the effective electro-mechanical property and history variable of the fibrous unit cell are:

$$
\begin{gathered}
\overline{\mathbf{O}}^{t}=\sum_{\alpha=1}^{\mathrm{IV}} c^{(\alpha)} \mathbf{O}^{(\alpha), t} \mathbf{B}^{(\alpha), t} . \\
\bar{\Xi}^{t-\Delta t}=\sum_{\alpha=1}^{\mathrm{IV}} c^{(\alpha)}\left(\mathbf{O}^{(\alpha), t} \mathbf{X}^{(\alpha), t}+\mathbf{\Xi}^{(\alpha), t-\Delta t}\right) .
\end{gathered}
$$

The linearized constitutive model for the fiber subcell I is obtained directly from Equation (22). The matrix subcells II, III, and IV in the fiber unit cell consist of piezoelectric fillers dispersed in the polymeric matrix. The electro-mechanical properties of these subcells are determined using the particle unit-cell model, comprising of eight subcells (Figure 2). The average field variables in the matrix subcells II, III, and IV are determined as:

$$
\boldsymbol{\Xi}^{(\alpha), t}=\sum_{\beta=1}^{8} c^{(\alpha, \beta)} \mathbf{\Xi}^{(\alpha, \beta), t}, \quad \alpha=\text { II, III, IV. }
$$

The superscript $(\alpha, \beta)$ indicates the subcells' numbers corresponding to the particulate unit cell $(\beta)$ and fiber unit cell $(\alpha)$. The particle volume fraction is defined as $c^{(\mathrm{II}, 1)}=V^{(\mathrm{II}, 1)} / V^{(\mathrm{II})}$ (volume fraction of the filler particles in the polymeric matrix) which should be the same as $c^{(\mathrm{III}, 1)}$ and $c^{(\mathrm{IV}, 1)}$. The corresponding particulate unit cell volumes are given by $V^{(\alpha)}=\sum_{\beta=1}^{\beta=8} V^{(\alpha, \beta)}$ with $\alpha=$ II, III, IV . The linearized constitutive relation for the particulate subcell $(\alpha, \beta)$ at current time $t$ is:

$$
\mathbf{\Xi}^{(\alpha, \beta), t}=\mathbf{O}^{(\alpha, \beta), t} \Delta \mathbf{T}^{(\alpha, \beta), t}+\mathbf{\Xi}^{(\alpha, \beta), t-\Delta t}, \quad \alpha=\mathrm{II}, \mathrm{III}, \mathrm{IV}, \quad \beta=1,2, \cdots, 8 .
$$

It is also necessary to determine the concentration matrix for the particulate unit-cell $\mathbf{B}^{(\alpha, \beta), t}$ and the vector of history variable $\mathbf{X}^{(\alpha, \beta), t}$ at current time $t$, which are defined through the relation:

$$
\Delta \mathbf{T}^{(\alpha, \beta), t}=\mathbf{B}^{(\alpha, \beta), t} \Delta \mathbf{T}^{(\alpha), t}+\mathbf{X}^{(\alpha, \beta), t}, \quad \alpha=\text { II, III, IV }, \quad \beta=1,2, \cdots, 8 .
$$

The above equation relates the incremental independent field variables of the matrix subcells II, III and IV to the corresponding incremental field variables of the particulate and polymer subcells. Substituting Equation (48) into Equation (47) and using the volume-average scheme in Equation (46), the corresponding dependent field variables for the matrix subcells are:

$$
\boldsymbol{\Xi}^{(\alpha), t}=\sum_{\beta=1}^{8} c^{(\alpha, \beta)} \mathbf{O}^{(\alpha, \beta), t} \mathbf{B}^{(\alpha, \beta), t} \Delta \mathbf{T}^{(\alpha), t}+\sum_{\beta=1}^{8} c^{(\alpha, \beta)}\left(\mathbf{O}^{(\alpha, \beta), t} \mathbf{X}^{(\alpha, \beta), t}+\mathbf{\Xi}^{(\alpha, \beta), t-\Delta t}\right), \quad \alpha=\text { II, III, IV } .
$$

Comparing Equation (49) to Equation (40) gives the overall electro-mechanical properties and history variables of the matrix subcells:

$$
\mathbf{O}^{(\alpha), t}=\sum_{\beta=1}^{8} c^{(\alpha, \beta)} \mathbf{O}^{(\alpha, \beta), t} \mathbf{B}^{(\alpha, \beta), t}, \quad \alpha=\mathrm{II}, \mathrm{III}, \mathrm{IV} .
$$




$$
\boldsymbol{\Xi}^{(\alpha), t-\Delta t}=\sum_{\beta=1}^{8} c^{(\alpha, \beta)}\left(\mathbf{O}^{(\alpha, \beta), t} \mathbf{X}^{(\alpha, \beta), t}+\mathbf{\Xi}^{(\alpha, \beta), t-\Delta t}\right), \quad \alpha=\text { II, III, IV. }
$$

Finally, in order to evaluate the concentration matrices and history variables $\mathbf{B}^{(\alpha), t}, \mathbf{B}^{(\alpha, \beta), t}$ and $\mathbf{X}^{(\alpha), t}$, $\mathbf{X}^{(\alpha, \beta), t}$ in the hybrid-unit-cell model it is necessary to use the constitutive relations for the piezoelectric and polymer constituents together with the linearized micromechanical relations from the fibrous unit cell and the particulate unit cells. The linearized micromechanical relations for the fibrous and particulate unit cells can be found in [25]. Because of the nonlinear constitutive relations for the constituents, the linearized micromechanical relations generally violate the overall nonlinear responses, which results in the following residual vector:

$$
\left\{\mathbf{R}^{t}\right\}=\underset{252 \times 1}{\left[\mathbf{P}^{t}\right]}\left\{\underset{252 \times 252}{\left.\Delta \mathbf{T}_{s}^{t}\right\}}-\underset{252 \times 1}{[\mathbf{Q}]}\left\{\underset{9 \times 1}{\left.\Delta \overline{\mathbf{T}}^{t}\right\}}\right\}+\underset{252 \times 1}{\left\{\mathbf{Y}^{t}\right\}},\right.
$$

where

$$
\left\{\Delta \mathbf{T}_{s}^{t}\right\}=\left\{\Delta \mathbf{T}^{(\mathrm{I}), t}, \cdots, \Delta \mathbf{T}^{(\mathrm{IV}), t}, \Delta \mathbf{T}^{(\mathrm{II}, 1), t}, \cdots, \Delta \mathbf{T}^{(\mathrm{II}, 8), t}, \Delta \mathbf{T}^{(\mathrm{III}, 1), t}, \cdots, \Delta \mathbf{T}^{(\mathrm{III}, 8), t}, \Delta \mathbf{T}^{(\mathrm{IV}, 1), t}, \cdots, \Delta \mathbf{T}^{(\mathrm{IV}, 8), t}\right\}^{\top},
$$

and $\left\{\mathbf{Y}^{t}\right\}$ results from the stress and electric field equilibrium conditions in the subcells, and the differences in the history variables from the displacement compatibility and electric displacement continuity at the interfaces between the adjacent subcells. $\left[\mathbf{P}^{t}\right]$ matrix is a function of the electric fields, material parameters and the volume fraction of each subcell at current time $t$ and the $[\mathbf{Q}]$ matrix is a constant matrix from the micromechanical relations. The dimension of each matrix is denoted on its bottom. A fixed-point iterative method is used to minimize the above residual vector at each time step. Once the residual vector has been minimized, the increment of the independent field variable in each subcell is given as:

$$
\left\{\Delta \mathbf{T}_{s}^{t}\right\}=\left[\mathbf{B}_{s}^{t}\right]\left\{\Delta \overline{\mathbf{T}}^{t}\right\}+\left\{\mathbf{X}_{s}^{t}\right\}
$$

where

$$
\begin{gathered}
{\left[\mathbf{B}_{s}^{t}\right]=\left[\mathbf{P}^{t}\right]^{-1}[\mathbf{Q}],} \\
\left\{\mathbf{X}_{s}^{t}\right\}=-\left[\mathbf{P}^{t}\right]^{-1}\left\{\mathbf{Y}^{t}\right\} .
\end{gathered}
$$

$\left[\mathbf{B}_{s}^{t}\right]$ comprises the elements of the concentration matrices $\mathbf{B}^{(\alpha), t}$ and $\mathbf{B}^{(\alpha, \beta), t}$, i.e.,

$$
\left[\mathbf{B}_{s}^{t}\right]=\left[\mathbf{B}^{(\mathrm{I}), t}, \cdots, \mathbf{B}^{(\mathrm{IV}), t}, \mathbf{B}^{(\mathrm{II}, 1), t}, \cdots, \mathbf{B}^{(\mathrm{II}, 8), t}, \mathbf{B}^{(\mathrm{III}, 1), t}, \cdots, \mathbf{B}^{(\mathrm{III}, 8), t}, \mathbf{B}^{(\mathrm{IV}, 1), t}, \cdots, \mathbf{B}^{(\mathrm{IV}, 8), t}\right]^{\top} .
$$

$\left\{\mathbf{X}_{s}^{t}\right\}$ includes the history variables $\mathbf{X}^{(\alpha), t}$ and $\mathbf{X}^{(\alpha, \beta), t}$, i.e.,

$$
\left\{\mathbf{X}_{s}^{t}\right\}=\left\{\mathbf{X}^{(\mathrm{I}), t}, \cdots, \mathbf{X}^{(\mathrm{IV}), t}, \mathbf{X}^{(\mathrm{II}, 1), t}, \cdots, \mathbf{X}^{(\mathrm{II}, 8), t}, \mathbf{X}^{(\mathrm{III}, 1), t}, \cdots, \mathbf{X}^{(\mathrm{III}, 8), t}, \mathbf{X}^{(\mathrm{IV}, 1), t}, \cdots, \mathbf{X}^{(\mathrm{IV}, 8), t}\right\}^{\top} .
$$

Once $\mathbf{B}^{(\alpha), t}, \mathbf{B}^{(\alpha, \beta), t}, \mathbf{X}^{(\alpha), t}$ and $\mathbf{X}^{(\alpha, \beta), t}$ have been determined, the effective electro-mechanical property $\overline{\mathbf{O}}^{t}$ and the field variable $\overline{\boldsymbol{\Xi}}^{t}$ are evaluated via Equations (44), (50) and (38), (42), (50), (51), respectively. It is noted that different incremental independent field variables, e.g., $(\Delta \boldsymbol{\varepsilon}, \Delta \mathbf{E})$ can be chosen to derive the hybridunit-cell model following a similar procedure.

\section{Numerical Implementation}

This section presents numerical analyses of the hybrid-unit-cell model. We first compare the predictions of the effective properties of hybrid composites with existing experimental data, which is limited to linear elastic moduli. We then conduct parametric studies on investigating the effects of constituent compositions, boundary conditions and loading history on the overall performance of hybrid piezocomposites.

\subsection{Comparison with Experimental Data}

Available experimental data for hybrid composites were primarily focused on the overall mechanical properties. The presented nonlinear hybrid-unit-cell model should be capable of predicting the overall properties of the hybrid composites without electro-mechanical coupling effect. Reference [5] reported the effective longitudinal 
Young's modulus of a hybrid composite with unidirectional carbon fibers dispersed in an alumina/epoxy matrix with the alumina particle volume fraction of 0.1. Figure 3(a) depicts the comparisons of the longitudinal elastic moduli of the hybrid composites and fiber reinforced polymer (FRP) composites obtained from the hybrid unit-cell model and experimental data. For the FRP composite, a hybrid unit-cell with zero percent particle volume content is considered. Adding particle to the polymeric matrix slightly improves the effective longitudinal moduli of the hybrid composite. Slight variation between the prediction and experimental data is observed, indicating that the micromechanics model give a reasonable predictions. Since [5] did not report the constituent properties, we calibrate the transverse and longitudinal moduli $\left(E_{22}\right.$ and $\left.E_{33}\right)$ for the carbon fiber and the modulus $(E)$ for the epoxy by using the experimental data on the FRP composite with the fiber volume fraction 0.41 shown in Figure 3(a) ${ }^{2}$. The constituent properties used in the simulation are listed in Table 5. The experimental data for the effective transverse moduli shown in Figure 3(b) were obtained from [40]. Using the fiber volume fraction 0.4 with the alumina particle volume fraction 0.1 shown in Figure 3(a) we further determine the elastic modulus of the alumina, which is $416 \mathrm{GPa}^{3}$. The calibrated material properties are then used to evaluate the effective longitudinal and transverse moduli of the hybrid composite with different fiber volume contents, as shown in Figure 3 (indicated by solid lines). It is seen that adding stiffer particles to the polymeric matrix can significantly enhance the transverse modulus. Reference [40] did not report the experimental data for the transverse moduli of the hybrid composite.

\subsection{Parametric Studies}

We first examine the effect of constituent compositions on the overall nonlinear electro-mechanical responses of a hybrid piezocomposite subjected to large electric fields but lower than coercive electric field (the constitutive relations in Equations (1) and (2) are used for polarized PZT fibers and particles). The matrix of the hybrid piezocomposite is first considered as elastic solid such as Araldite D while the polarized PZT-G1195 is used for the inhomogeneities ${ }^{4}$. The material properties of the Araldite D and polarized PZT-G1195 used for simulations reported in [42] and [25], respectively.

Figure 4 shows the effective transverse stress $\bar{\sigma}_{11}$ and longitudinal stress $\bar{\sigma}_{33}$ due to an applied electric field $\bar{E}_{3}$ along the poling direction, which is the longitudinal fiber direction ( $x_{3}$ axis) up to $1 \mathrm{MV} / \mathrm{m}^{5}$ for a fully constrained displacement of the PZT-G1195/[PZT-G1195/Araldite D] hybrid piezocomposite with PZT-G1195 fiber volume fraction $(\mathrm{VF})=0.4$ and several PZT-G1195 particle VFs $=0-0.5$. The linear response for the composite with zero content of PZT-G1195 fillers is also shown for comparison. Figure 4(a) shows that as the filler VF increases the effective transverse stress $\bar{\sigma}_{11}$ is significantly enhanced while the effective longitudinal stress $\bar{\sigma}_{33}$ is insensitive to the existence of piezoelectric fillers even for higher particle contents, as shown in Figure 4(b). This is due to the fact that the transverse stress $\bar{\sigma}_{11}$ is a matrix-dominated response and high stiffness of the PZT-G1195 fillers increases the stiffness of the overall matrix. In contrast, the longitudinal stress $\bar{\sigma}_{33}$ is a fiber-dominated response and insignificant improvements in the longitudinal properties are shown with adding PZT-G1195 fillers. Thus, dispersing stiffer fillers into a softer matrix in a fibrous piezocomposite will be useful for improving the blocked stress for the 3 - 1 operating mode.

We also consider a stress free boundary condition for a PZT-G1195/[PZT-G1195/Araldite D] hybrid piezocomposite with PZT-G1195 fiber VF $=0.4$ and PZT-G1195 particle VF varies from 0 to 0.5 , subjected to an applied electric field $\bar{E}_{3}$ along the poling direction up to $1 \mathrm{MV} / \mathrm{m}$. Figure 5 depicts the effective transverse strain $\bar{\varepsilon}_{11}$ and longitudinal strain $\bar{\varepsilon}_{33}$. The absolute values of the effective strains $\bar{\varepsilon}_{11}$ and $\bar{\varepsilon}_{33}$ both decrease as the PZT-G1195 fillers increase. This is because adding PZT-G1195 particles in the matrix increases the stiffness of the matrix and leads to a stiffer hybrid piezocomposite, which causes less actuation strains under the same electric field input. It is known that in piezoelectric materials larger blocked stresses are accompanied by smaller

\footnotetext{
${ }^{2}$ For the calibration procedure, first we assume Poisson ratios $v_{31}=0.2, v_{12}=0.25$, longitudinal shear modulus $G_{31}=28$ GPa for the carbon fiber which are referred to [39], and Poisson ration $v=0.35$ for the epoxy resin which is referred to [20].

${ }^{3}$ Experimental examination from [41] shown that the elastic modulus of the alumina is between 338 and $416 \mathrm{GPa}$.

${ }^{4}$ The piezoelectric inhomogeneities (both fibers and parties) of a hybrid piezocomposite only can be polarized after it forms a hybrid composite in order to ensure that all piezoelectric inhomogeneities are poled along the same direction ( $x_{3}$ direction in our study.) Electrodes are placed on the each end of fibers in order to generate the electric field along with the $x_{3}$ direction (fiber direction). Thus, both PZT particles and fibers will experience the electric field along its polarized direction, which induces extension or shrink along with their polarized direction (the $x_{3}$ direction.)

${ }^{5} \mathrm{MV} / \mathrm{m}$ is $85 \%$ of the coercive electric field of the polarized PZT-G1195 and thus the polarized PZT-G1195 does not experience depolarization and polarization switching reported by [32].
} 

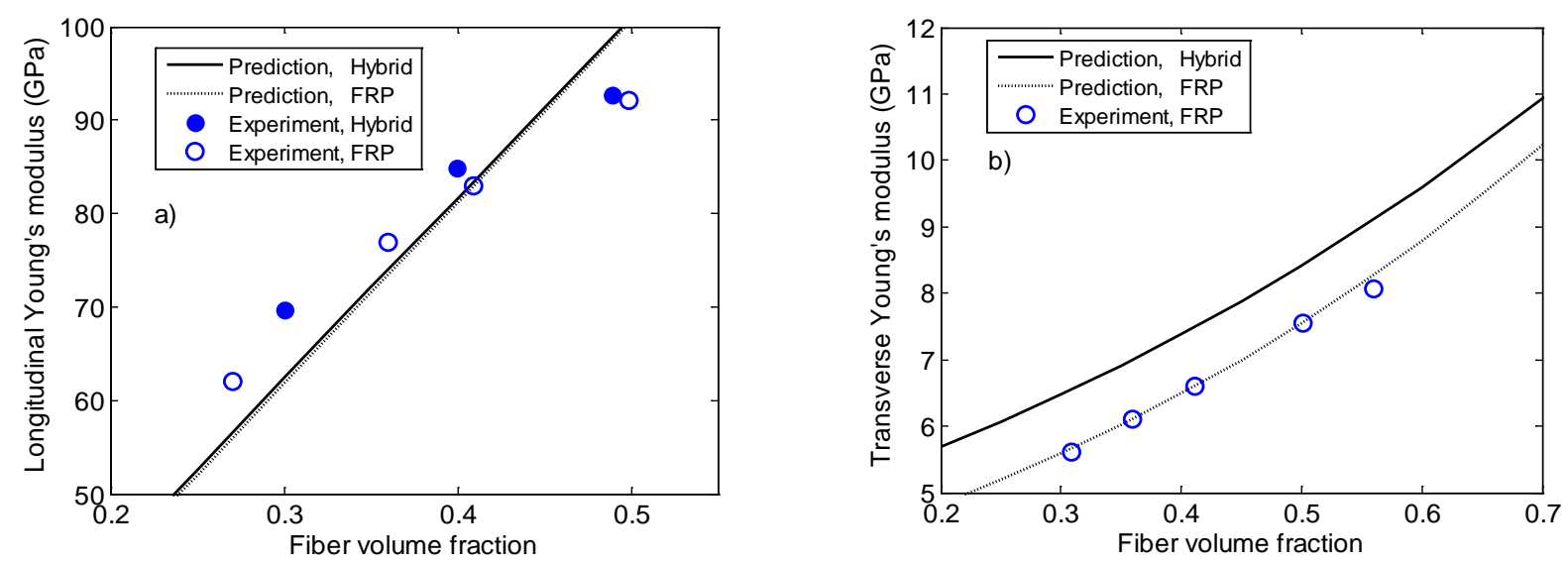

Figure 3. Comparison of the micromechanical predictions to experimental data, (a) [5] and (b) [40], for the effective longitudinal elastic moduli for the hybrid (solid lines) and FRP (dotted lines) composites as a function of fiber volume fraction. The hybrid composite is formed by carbon fibers embedded in the epoxy matrix which is reinforced by 0.1 volume fraction of alumina particles.
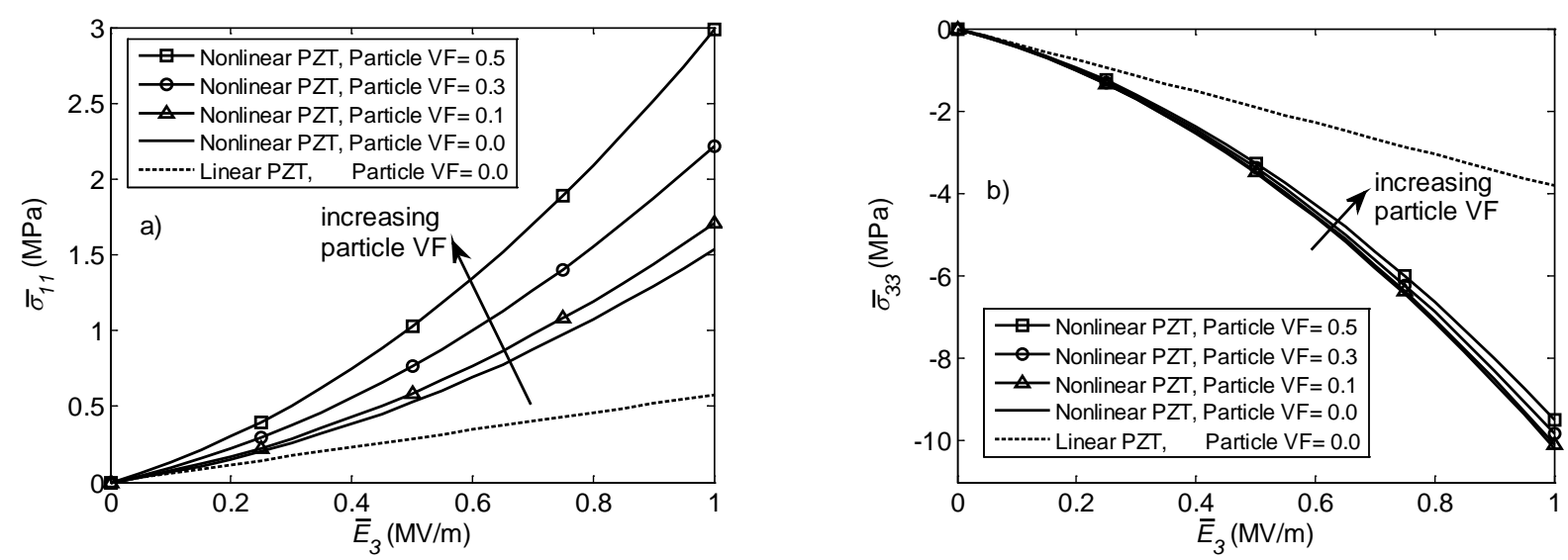

Figure 4. Effective (a) transverse stress $\bar{\sigma}_{11}$ and (b) longitudinal stress $\bar{\sigma}_{33}$ responses for the fully constrained displacement of the PZT-G1195/[PZT-G1195/Araldite D] hybrid piezocomposite with a fixed PZT-G1195 fiber VF = 0.4 and various PZT-G1195 particle VFs, 0.0, 0.1, 0.3 and 0.5, due to an applied electric field $\bar{E}_{3}$ along the poling direction.
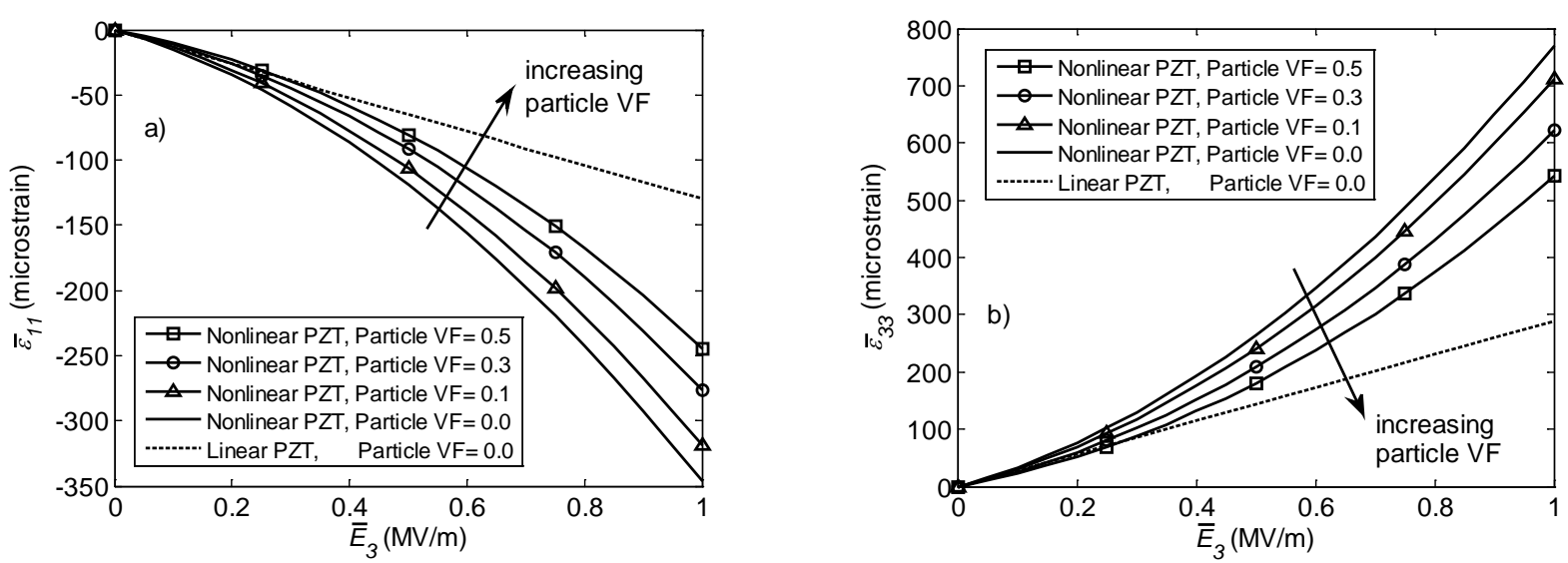

Figure 5. Effective (a) transverse strain $\bar{\varepsilon}_{11}$ and (b) longitudinal strain $\bar{\varepsilon}_{33}$ responses for the stress free PZT-G1195/ [PZT-G1195/Araldite D] hybrid piezocomposite with a fixed PZT-G1195 fiber VF $=0.4$ and various PZT-G1195 particle VFs, 0.0, 0.1, 0.3 and 0.5 , due to an applied electric field $\bar{E}_{3}$ along the poling direction. 
free strains, and vice versa. Adding stiffer fillers, i.e., PZTs, into a relatively soft matrix, i.e., polymer, in a fiberreinforced piezocomposite is done to improve the transverse blocked stress.

In order to study the time-dependent responses due a viscoelastic constituent in a hybrid piezocomposite, FM73 polymer whose dielectric constants are taken as $\kappa_{11}=\kappa_{22}=\kappa_{33}=0.039 \times 10^{-9} \mathrm{~F} / \mathrm{m}$ is used for the polymer constituent and its viscoelastic properties are given in Table 6. Fully constrained PZT-G1195/[PZT-G1195/FM73 polymer] hybrid piezocomposites with PZT-G1195 fiber VF $=0.4$ and PZT-G1195 particle filler VFs $=0$ and 0.5 are subjected to a cyclic electric field, $\bar{E}_{3}(t)=-0.5 \cos (2 \pi f t)+0.5 \mathrm{MV} / \mathrm{m}$ along the poling direction with various frequencies $f=0.5,1$ and $10 \mathrm{~Hz}$. The response of the effective transverse stress $\bar{\sigma}_{11}$ amplitude (maximum stress) as a function of number of cycles at different loading frequencies is shown in Figure 6. As the number of cycles increase (longer duration of loading), the stress amplitude decreases until it reaches steady value, i.e., fully relaxed stress state. Higher frequency leads to more cycle needed to reach steady state, which is expected since slow input would give enough time for the viscoelastic polymers to experience stress relaxation. The hybrid piezocomposite (Figure 6(a)) and the fiber-reinforced piezocomposite (Figure 6(b)) experience the same trends under cyclic loading with higher effective blocked stress $\bar{\sigma}_{11}$ in the hybrid piezocomposite.

Next, we investigate the overall hysteretic polarization switching and butterfly strain responses of a hybrid piezocomposite with various constituent compositions and under different loading histories. The constitutive relations in Equations (3) and (4) are used for polarization switching response of PZT-51 fibers and particles. The matrix of the hybrid piezocomposite is considered as FM73 polymer.

Figure 7 depicts steady state electric displacement and longitudinal strain responses of a stress free boundary condition for a PZT-51/[PZT-51/FM73 polymer] hybrid piezocomposite with PZT-51 fiber VF $=0.4$ and PZT-51 particle VFs $=0.0,0.2$ and 0.4 , subjected to a cyclic electric loading $\bar{E}_{3}=1.2 \sin (2 \pi f t) \mathrm{MV} / \mathrm{m}$ along

Table 5. Mechanical properties of the carbon fiber, epoxy and alumina (The material properties are determined from [5] and [40]).

\begin{tabular}{|ccc|}
\hline & Carbon fiber \\
\hline Longitudinal Young's modulus, $E_{33}(\mathrm{GPa})$ & 198 & \\
Transverse Young's modulus, $E_{22}(\mathrm{GPa})$ & 16 & \\
Major Poisson's ratio, $v_{31}$ & 0.20 & \\
In-plane Poisson's ratio, $v_{12}$ & 0.25 & \\
\hline Longitudinal shear modulus, $G_{31}(\mathrm{GPa})$ & 28 & Alumina \\
\hline & Epoxy & 416 \\
\hline Young's modulus, $E(\mathrm{GPa})$ & 3.4 & 0.23 \\
\hline
\end{tabular}

Table 6. Time-dependent compliance, instantaneous time-dependent (elastic) compliance and Poisson's ratio for the viscoelastic FM73 polymer ([43]).

\begin{tabular}{ccc}
\hline$n^{\mathrm{a}}$ & $\lambda_{n}\left(\mathrm{sec}^{-1}\right)$ & $D_{n}\left(\mathrm{GPa}^{-1}\right)$ \\
\hline 1 & 1 & 0.0210 \\
2 & $10^{-1}$ & 0.0216 \\
& $D_{0}=0.369\left(\mathrm{GPa}^{-1}\right)$ & \\
& $v=0.35$ & \\
\hline
\end{tabular}

${ }^{\mathrm{a}}$ We only consider the first two terms of the series of exponential functions to the viscoelastic FM73 polymer. This simplification will not affect us to qualitatively understand the influence of the viscoelastic constituent to the overall responses of composites but it will dramatically reduce computational cost. 


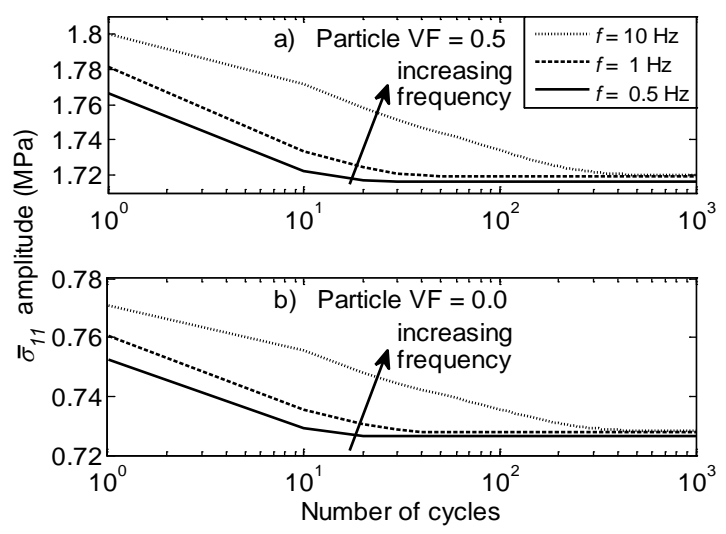

Figure 6. Effective transverse stress $\bar{\sigma}_{11}$ amplitude vs. number of cyclers for the fully constrained displacement of the PZT-G1195/[PZT-G1195/FM73 polymer] hybrid piezocomposite with PZT-G1195 fiber VF $=0.4$ and various PZT-G1195 particle VFs, (a) 0.0 and (b) 0.5 , due to a cyclic electric field $\bar{E}_{3}(t)=-0.5 \cos (2 \pi f t)+0.5$ $\mathrm{MV} / \mathrm{m}$ with various frequencies $f=0.5,1$ and $10 \mathrm{~Hz}$ along the poling direction (Logarithmic scale on the horizontal axis).

the longitudinal fiber direction ( $x_{3}$ direction) with the frequency $f=1 \mathrm{~Hz}$. It is expected that the heights of the butterfly curves (Figure 7(b), Figure 7(d), Figure 7(f)) of the hybrid piezocomposite decrease as PZT-51 particles increase because PZT-51 fillers increase the overall stiffness of the matrix. In contrast, the polarization responses (Figure 7(a), Figure 7(c), Figure 7(e)) are only slightly influenced by the adding the active fillers since the response is dominated by the fibers. At the saturated (steady state) condition, the strains in the butterfly curves at the coercive electric field limit are slightly higher than zero, which are due to the time-dependent PZT-51 and FM73 polymer materials. Even though the hybrid composites are under stress-free boundary conditions, the heterogeneity in the composites leads to existence of internal stresses when electric field is applied. Several discontinuities in the hysteretic polarization and butterfly curves are observed in Figure 7(g) and Figure 7(h), respectively, when PZT-51 particle VF increases to 0.55 . These discontinuities occur when the magnitude of compressive stress $\sigma_{33}^{t}$ in the PZT-51 fiber exceeds the coercive stress limit ( $\sigma_{c}=25 \mathrm{MPa}$ for PZT-51) either from $\sigma_{33}^{t}>-\sigma_{c}$ to $\sigma_{33}^{t} \leq-\sigma_{c}$ or from $\sigma_{33}^{t} \leq-\sigma_{c}$ back to $\sigma_{33}^{t}>-\sigma_{c}$. When the compressive stress $\sigma_{33}^{t}$ in the PZT-51 fiber is greater than the coercive stress limit, polarization switching occurs, whose effect is incorporated in Equations (13) and (14). Changes in the material parameters, when a compressive stress is higher than the coercive stress limit, lead to discontinuities in the electro-mechanical responses. This issue has been discussed in [35] for homogeneous ferroelectric ceramics.

Next, we examine the effect of prescribing compressive stresses on the overall nonlinear rate-dependent hysteretic electro-mechanical responses of a PZT-51/[PZT-51/FM73 polymer] hybrid piezocomposite with PZT-51 fiber VF $=0.4$ and PZT-51 particle VF $=0.2$, subjected to a cyclic electric loading $\bar{E}_{3}=1.2 \sin (2 \pi f t) \mathrm{MV} / \mathrm{m}$ along the fiber direction with the frequency $f=1 \mathrm{~Hz}$ and constant compressive stresses $\bar{\sigma}_{33}=0,-15$ and -30 MPa. The coercive electric field changes with the compressive stress, which for the studied PZT-51 is described as:

$$
E_{c}= \begin{cases}E_{c}^{0}-0.0041\left|\sigma_{33}^{t}\right|, & \sigma_{33}^{t}<0, \\ E_{c}^{0}, & \sigma_{33}^{t} \geq 0 .\end{cases}
$$

Figure 8 shows the steady state electric displacement and longitudinal strain responses after 100 cycles. The compressive stresses limit the amount of polarization to be generated from electric field inputs, as a result smaller hysteretic polarization and butterfly strain curves are observed when higher compressive stress is applied.

We also study the effect of frequencies on the overall hysteretic electro-mechanical responses of a hybrid piezocomposite. We consider a stress free PZT-51/[PZT-51/FM73 polymer] hybrid piezocomposite with PZT-51 fiber VF $=0.4$ and PZT-51 particle VF $=0.2$ subjected to cyclic electric loadings $\bar{E}_{3}=1.2 \sin (2 \pi f t)$ 

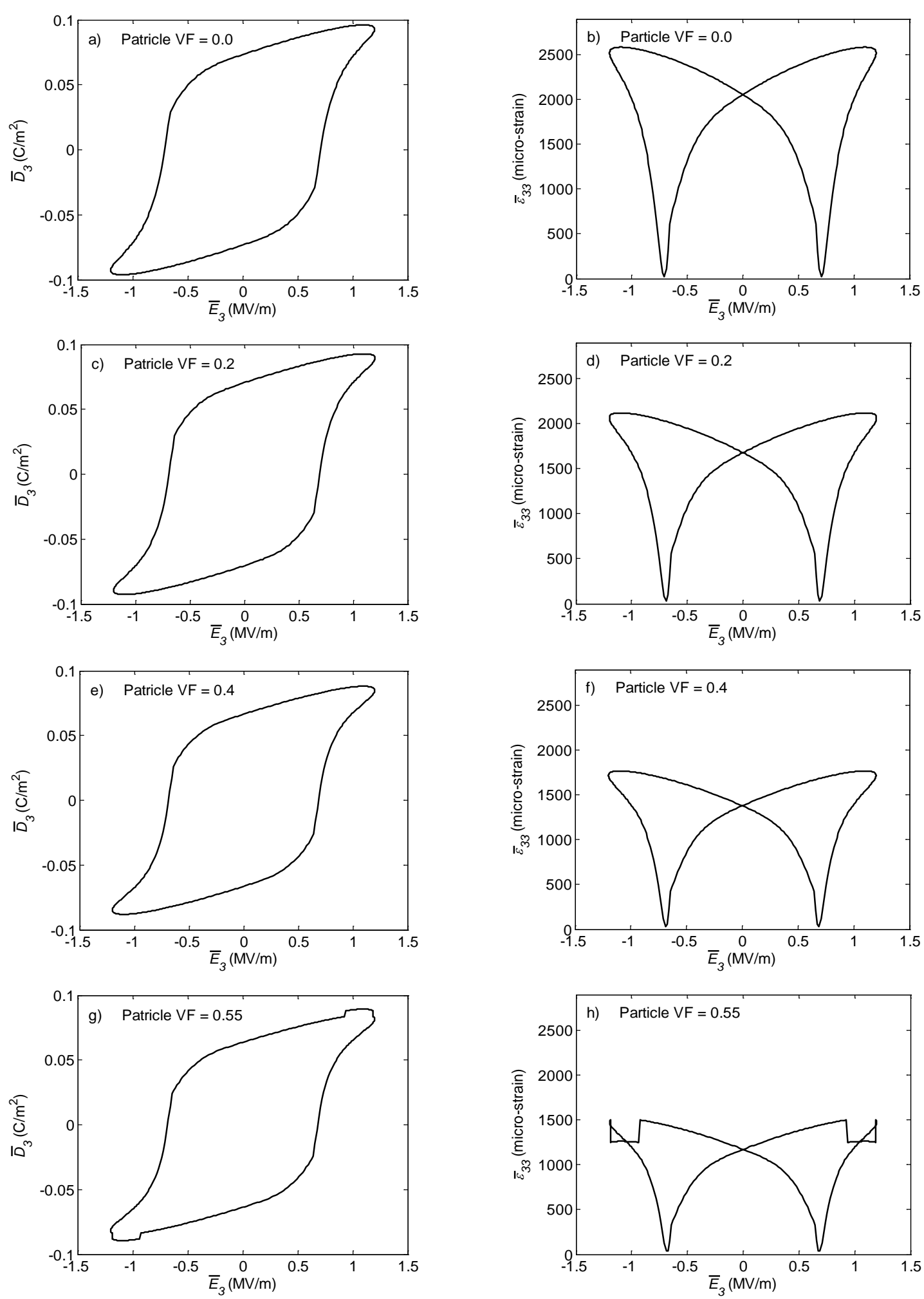

Figure 7. Effective (a), (c), (e), (g) electric displacement $\bar{D}_{3}$ and (b), (d), (f), (h) longitudinal strain $\bar{\varepsilon}_{33}$ responses for the stress free PZT-51/[PZT-51/FM73 polymer] hybrid piezocomposite with a fixed PZT-51 fiber VF $=0.4$ and various PZT-51 particle VFs, (a), (b) 0.0; (c), (d) 0.2; (e), (f) 0.4; (g), (h) 0.55, due to a cyclic electric loading $\bar{E}_{3}(t)=1.2 \sin (2 \pi f t)$ $\mathrm{MV} / \mathrm{m}$ with frequency $f=1 \mathrm{~Hz}$ along the poling direction. 100 cycles are enough to reach steady state. 

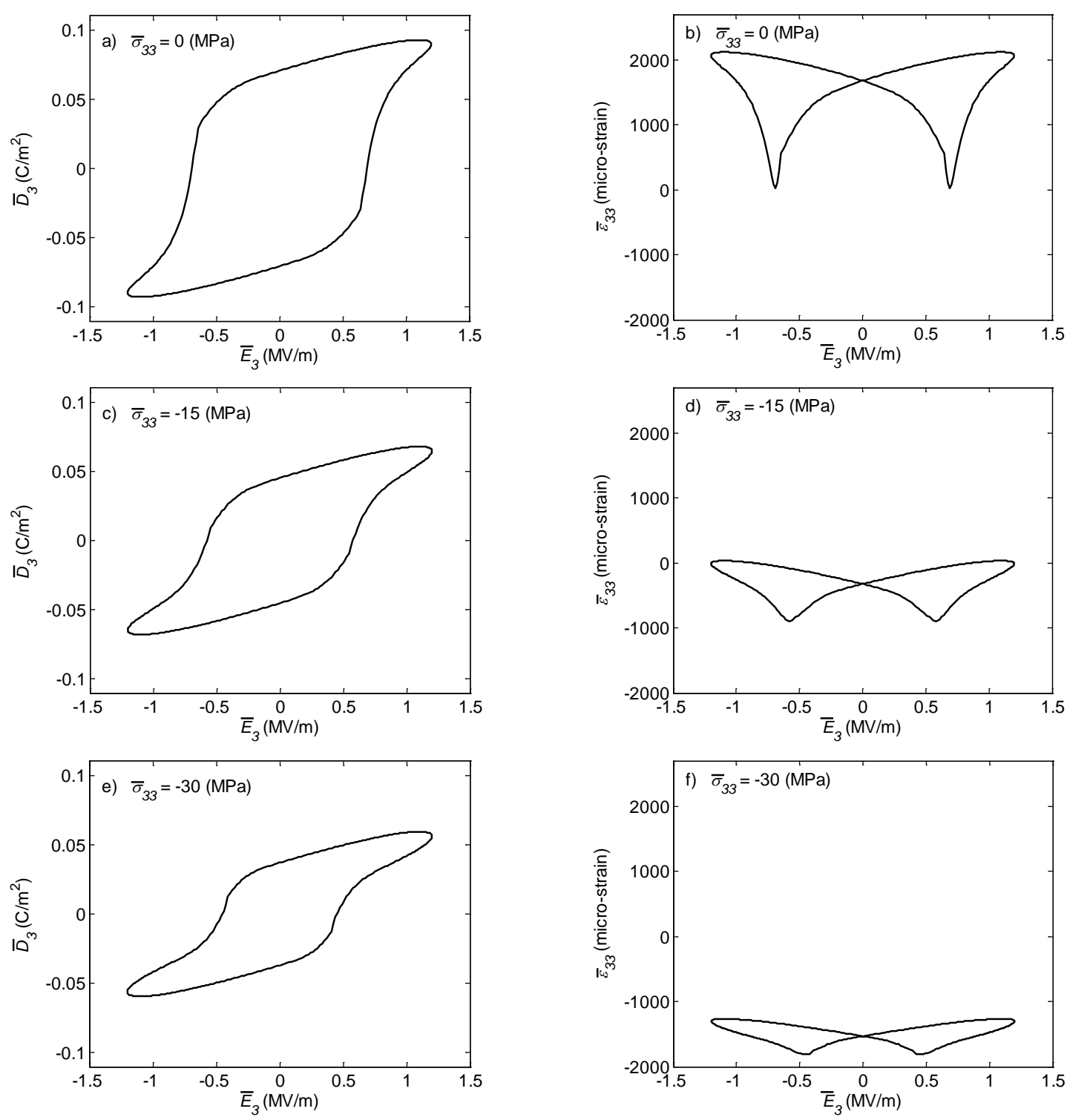

Figure 8. Effective (a), (c), (e) electric displacement $\bar{D}_{3}$ and (b), (d), (f) longitudinal strain $\bar{\varepsilon}_{33}$ responses for the PZT-51/[PZT-51/FM73 polymer] hybrid piezocomposite with PZT-51 fiber VF $=0.4$ and PZT-51 particle VF $=0.2$ subjected to both a cyclic electric loading $\bar{E}_{3}(t)=1.2 \sin (2 \pi f t) \quad \mathrm{MV} / \mathrm{m}$ with frequency $f=1 \mathrm{~Hz}$ along the poling direction and various constant mechanical stresses $\bar{\sigma}_{33}$, (a), (b) 0; (c), (d) -15 ; (e), (f) $-30 \mathrm{MPa} .100$ cycles are enough to reach steady state.

along the fiber axis with different frequencies $f=0.5,1$ and $10 \mathrm{~Hz}$. Figure 9 depicts the responses of the polarization and longitudinal strain for the first six cycles. Lower frequency loading leads to larger hysteretic response since slower loading allows for the materials to experience more pronounced time-dependent response. In this analysis, PZT fibers and particles experiences relaxation-like polarization response while the matrix exhibits viscoelastic deformation. For the higher frequency loading, smaller hysteretic responses are seen and saturated (steady-state) condition is reached after a first few cycle, indicating negligible time-dependent response. For high frequency loading cases, the hysteretic response is mainly due to the irreversible polarization during polarization switching. We then examine the evolution of the amplitude of the effective strain of the butterfly strain response at various cycles. A PZT-51/[PZT-51/FM73 polymer] hybrid piezocomposite with PZT-51 fiber VF = 0.4 and PZT-51 particle VF $=0.2$ under a cyclic electric loading $\bar{E}_{3}(t)=1.2 \sin (2 \pi f t) \mathrm{MV} / \mathrm{m}$ with frequency 

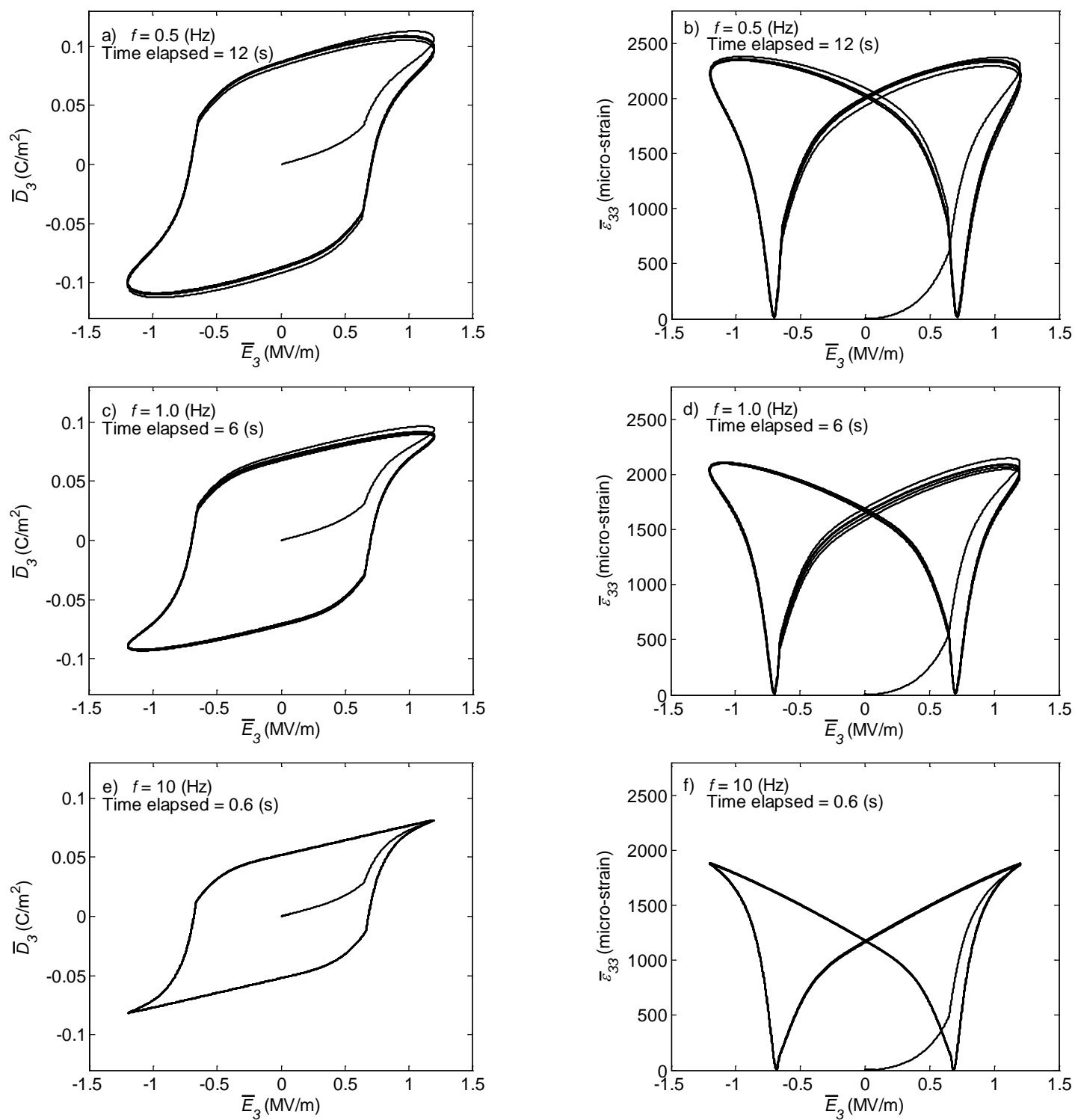

Figure 9. Effective (a), (c), (e) electric displacement $\bar{D}_{3}$ and (b), (d), (f) longitudinal strain $\bar{\varepsilon}_{33}$ responses for the stress free PZT-51/[PZT-51/FM73 polymer] hybrid piezocomposite with PZT-51 fiber VF $=0.4$ and PZT-51 particle VF $=0.2$ subjected to a cyclic electric loading $\bar{E}_{3}(t)=1.2 \sin (2 \pi f t) \quad \mathrm{MV} / \mathrm{m}$ with various frequencies $f$, (a), (b) 0.5 ; (c), (d) 1 ; (e), (f) $10 \mathrm{~Hz}$, along the poling direction. First six cycles are plotted.

$f=1 \mathrm{~Hz}$ along the poling direction is used in the analysis. Figure 10 depicts the normalized effective strain amplitude $^{6}$ at various cycles. The initial drop in the normalized effective strain amplitude is due to time-dependent polarization effect in the PZT-51 fibers and then the strain amplitude increases at later cycles because of the creep deformation effect in the FM73 polymer constituent. For further explanation, it is seen in Figure 11(a) that the strain amplitude in the PZT-51 constituent under cyclic electric field decreases before reaching steady state, while the strain amplitude in the FM73 polymermatrix constituent (Figure 11(b)) under cyclic stress input increases with increasing number of cycles. The different responses in the PZT-51 and FM73 polymer leads to complex hysteretic responses of the hybrid composites and higher number of cycles is required to reach steady state.

${ }^{6}$ The normalized strain $\bar{\varepsilon}_{33}$ amplitude is normalized with respect to the maximum strain $\bar{\varepsilon}_{33}$ in the first cycle. 


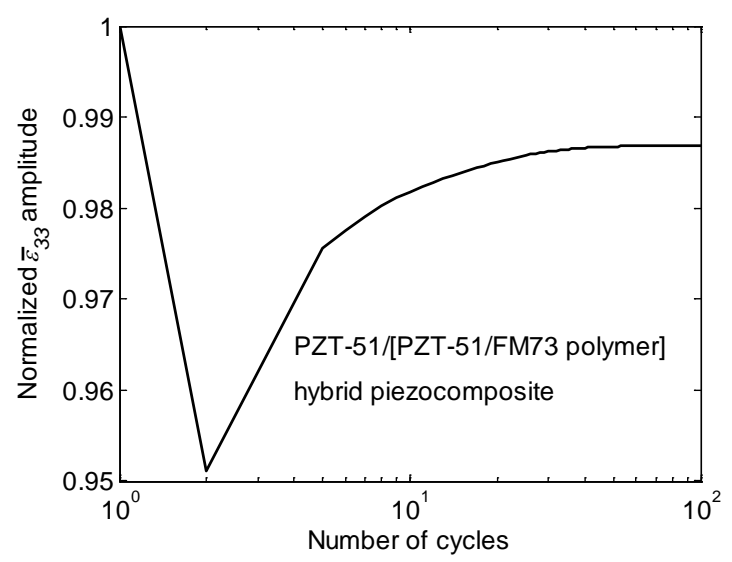

Figure 10. Normalized effective longitudinal strain $\bar{\varepsilon}_{33}$ amplitude vs. number of cyclers for the stress free PZT-51/[PZT-51/FM73 polymer] hybrid piezocomposite with PZT-51 fiber VF $=0.4$ and PZT-51 particle VF $=0.2$ due to a cyclic electric loading $\bar{E}_{3}(t)=1.2 \sin (2 \pi f t) \quad \mathrm{MV} / \mathrm{m}$ with frequency $f=1 \mathrm{~Hz}$ along the poling direction. (Logarithmic scale on the horizontal axis).
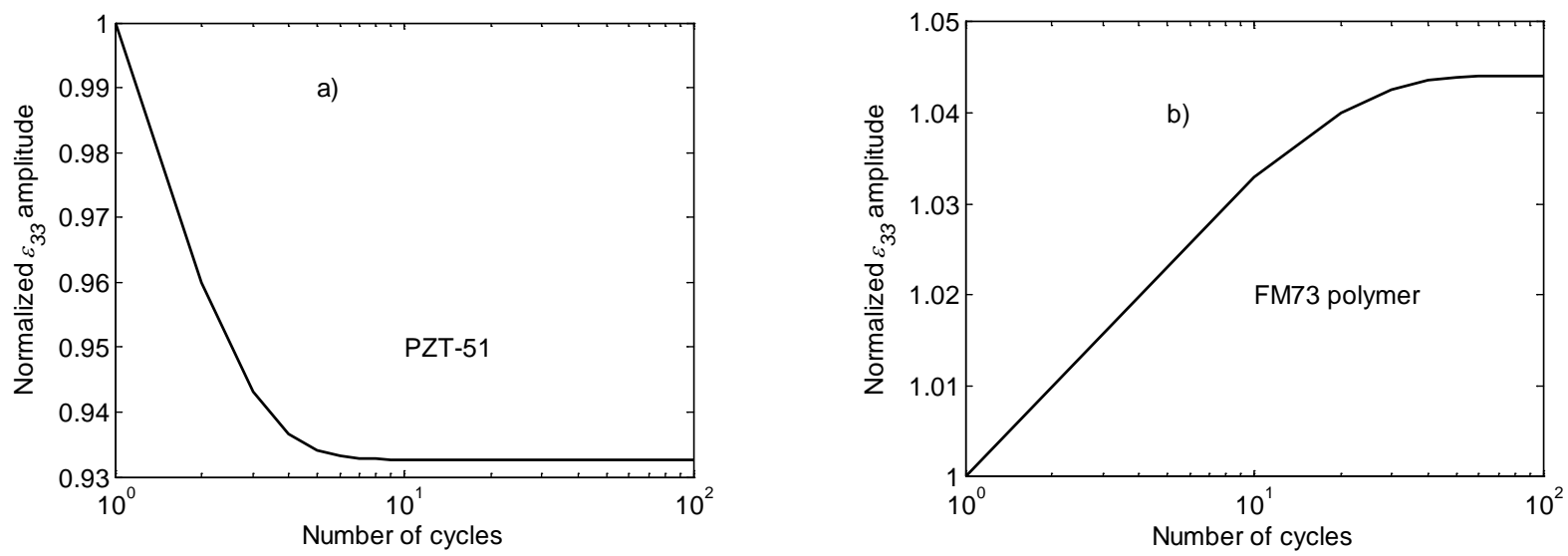

Figure 11. Normalized strain amplitude vs. number of cyclers. (a) Pure PZT-51 subject to a cyclic electric loading with frequency $f=1 \mathrm{~Hz}$ along the poling direction. (b) Pure FM73 polymer subject to a cyclic mechanical loading with frequency $f=1 \mathrm{~Hz}$. (Logarithmic scale on the horizontal axis).

\section{Conclusions}

We have developed a hybrid-unit-cell model for predicting the effective nonlinear and rate-dependent hysteretic responses of active hybrid composites. The studied hybrid piezocomposites consist of unidirectional piezoelectric fibers embedded in a polymeric matrix, which is reinforced with piezoelectric particles. Nonlinear electromechanical constitutive models, including polarization switching response, are used for the active fibers and particles, while a viscoelastic solid-like model is used for the polymer. In order to predict the effective nonlinear rate-dependent electro-mechanical responses, linearized micromechanical relations are first imposed in order to provide trial solutions at each instant of time. An iterative scheme, i.e., fixed-point method, is then added to minimize errors from linearizing the nonlinear electro-mechanical and time-dependent responses.

We have performed several analyses on understanding the nonlinear electro-mechanical responses of hybrid piezocomposites using the above hybrid-unit-cell model. The results are summarized as follow: The hybrid unit-cell model is capable of capturing the linear elastic response of fiber-reinforced composites and hybrid composites, which are tested with limited experimental data. Adding PZT fillers significantly improve the blocked stress in the transverse fiber direction while insignificantly affects the overall electro-mechanical performance in the longitudinal fiber direction. This is because the matrix, whose properties change with adding the 
PZT fillers and dominate the transverse response. The free strains, however, significantly decrease in both transverse and longitudinal fiber directions as the amount of PZT fillers increase. This is due to the fact that adding stiffer PZT particles in a softer epoxy matrix results in a stiffer overall matrix. Thus, adding PZT fillers is useful for improving the blocked stress for active composites with 3 - 1 operating mode. Responses of the hybrid piezocomposites under cyclic electric field, with amplitude higher than the coercive electric field limit of the materials, and compressive stress loadings have been studied. Adding PZT fillers slightly reduces the hysteretic polarization response, and significantly decreases the hysteretic strain response. As the matrix becomes stiffer, matrix would experience smaller deformations when an electric field input is applied, resulting in smaller residual stresses ${ }^{7}$ in both fibers and matrix. Although its effect is minimum, the residual stress would affect the overall hysteretic polarization in composites. As also expected compressive stresses applied along the direction of electric field reduce the polarization capability of the composites. We also investigate the effect of frequencies on the overall electro-mechanical responses of hybrid composites. A lower frequency input allows the hybrid piezocomposites to undergo more pronounced time-dependent response, which in this case is shown by broader hysteretic responses. The hysteretic response indicates amount of energy being dissipated, which is converted into heat. It is noted that many applications of active materials would involve cyclic electro-mechanical loading, thus the hysteretic response can eventually lead to cyclic failures.

\section{Acknowledgements}

This research is sponsored by the National Science Foundation (NSF) under grant CMMI-1437086.

\section{References}

[1] Safari, A. (1994) Development of Piezoelectric Composites for Transducers. Journal de Physique III, 4, 1129-1149. http://dx.doi.org/10.1051/jp3:1994191

[2] Bent, A.A. and Hagood, N.W. (1997) Piezoelectric Fiber Composites with Interdigitated Electrodes. Journal of Intelligent Material Systems and Structures, 8, 903-919. http://dx.doi.org/10.1177/1045389X9700801101

[3] Gadkaree, K.P. (1992) Particulate-Fibre-Reinforced Glass Matrix Hybrid Composite. Journal of Materials Science, 27, 3827-3834. http://dx.doi.org/10.1007/BF00545465

[4] Tsai, J.-L. and Wu, M.-D. (2007) Organoclay Effect on Mechanical Responses of Glass/Epoxy Nanocomposites. Journal of Composite Materials, 41, 2513-2528. http://dx.doi.org/10.1177/0021998307076491

[5] Hussain, M., Nakahira, A. and Niihara, K. (1996) Mechanical Property Improvement of Carbon Fiber Reinforced Epoxy Composites by $\mathrm{Al}_{2} \mathrm{O}_{3}$ Filler Dispersion. Materials Letters, 26, 185-191. http://dx.doi.org/10.1016/0167-577X(95)00224-3

[6] Wang, S., Adanur, S. and Jang, Z. (1997) Mechanical and Thermo-Mechanical Failure Mechanism Analysis of Fiber/Filler Reinforced Phenolic Matrix Composites. Composites Part B: Engineering, 28, 215-231. http://dx.doi.org/10.1016/S1359-8368(96)00042-X

[7] Asi, O. (2009) Mechanical Properties of Glass-Fiber Reinforced Epoxy Composites Filled with $\mathrm{Al}_{2} \mathrm{O}_{3}$ Particles. Journal of Reinforced Plastics and Composites, 28, 2861-2867. http://dx.doi.org/10.1177/0731684408093975

[8] Vlasveld, D.P.N., Bersee, H.E.N. and Picken, S.J. (2005) Nanocomposite Matrix for Increased Fibre Composite Strength. Polymer, 46, 10269-10278. http://dx.doi.org/10.1016/j.polymer.2005.08.003

[9] Zheng, Y., Ning, R. and Zheng, Y. (2005) Study of $\mathrm{SiO}_{2}$ Nanoparticles on the Improved Performance of Epoxy and Fiber Composites. Journal of Reinforced Plastics and Composites, 24, 223-233. http://dx.doi.org/10.1177/0731684405043552

[10] Subramaniyan, A.K. and Sun, C.T. (2006) Enhancing Compressive Strength of Unidirectional Polymeric Composites Using Nanoclay. Composites Part A: Applied Science and Manufacturing, 37, 2257-2268.

[11] Cho, J., Chen, J.Y. and Daniel, I.M. (2007) Mechanical Enhancement of Carbon Fiber/Epoxy Composites by Graphite Nanoplatelet Reinforcement. Scripta Materialia, 56, 685-688. http://dx.doi.org/10.1016/j.scriptamat.2006.12.038

[12] Uddin, M.F. and Sun, C.T. (2008) Strength of Unidirectional Glass/Epoxy Composite with Silica Nanoparticle-Enhanced Matrix. Composites Science and Technology, 68, 1637-1643. http://dx.doi.org/10.1016/j.compscitech.2008.02.026

\footnotetext{
${ }^{7}$ The residual stress is defined as internal stresses in the constituents of the composites in absence of external mechanical stimuli. In this study, the residual stresses arise due to the mismatches in the properties and responses of the constituents when the composites are subjected to electric field input.
} 
[13] Asi, O. (2010) An Experimental Study on the Bearing Strength Behavior of $\mathrm{Al}_{2} \mathrm{O}_{3}$ Particle Filled Glass Fiber Reinforced Epoxy Composites Pinned Joints. Composite Structures, 92, 354-363. http://dx.doi.org/10.1016/j.compstruct.2009.08.014

[14] Hagood, N.W. and Bent, A.A. (1993) Development of Piezoelectric Fiber Composites for Structural Actuation. Proceedings of 34th AIAA Structures, Structural Dynamics, and Materials Conference, La Jolla, 19-22 April 1993, AIAA Paper No. 93-1717, 3625-3638. http://dx.doi.org/10.2514/6.1993-1717

[15] Bent, A.A., Hagood, N.W. and Rodgers, J.P. (1995) Anisotropic Actuation with Piezoelectric Fiber Composites. Journal of Intelligent Material Systems and Structures, 6, 338-349. http://dx.doi.org/10.1177/1045389X9500600305

[16] Newnham, R.E., Bowen, L.J., Klicker, K.A. and Cross, L.E. (1980) Composite Piezoelectric Transducers. Materials \& Design, 2, 93-106.

[17] Banno, H. (1983) Recent Developments of Piezoelectric Ceramic Products and Composites of Synthetic Rubber and Piezoelectric Ceramic Particles. Ferroelectrics, 50, 3-12. http://dx.doi.org/10.1080/00150198308014425

[18] Smith, W.A. and Auld, B.A. (1991) Modeling 1-3 Composite Piezoelectrics: Thickness-Mode Oscillations. IEEE Transactions on Ultrasonics, Ferroelectrics, and Frequency Control, 38, 40-47. http://dx.doi.org/10.1109/58.67833

[19] Nan, C.-W. and Jin, F.-S. (1993) Multiple-Scattering Approach to Effective Properties of Piezoelectric Composites. Physical Review B, 48, 8578-8582. http://dx.doi.org/10.1103/PhysRevB.48.8578

[20] Dunn, M.L. and Taya, M. (1993) Micromechanics Predictions of the Effective Electroelastic Moduli of Piezoelectric Composites. International Journal of Solids and Structures, 30, 161-175. http://dx.doi.org/10.1016/0020-7683(93)90058-F

[21] Aboudi, J. (1998) Micromechanical Prediction of the Effective Coefficients of Thermo-Piezoelectric Multiphase Composites. Journal of Intelligent Material Systems and Structures, 9, 713-722. http://dx.doi.org/10.1177/1045389X9800900903

[22] Odegard, G.M. (2004) Constitutive Modeling of Piezoelectric Polymer Composites. Acta Materialia, 52, 5315-5330. http://dx.doi.org/10.1016/j.actamat.2004.07.037

[23] Tan, P. and Tong, L. (2001) Micro-Electromechanics Models for Piezoelectric-Fiber-Reinforced Composite Materials. Composites Science and Technology, 61, 759-769. http://dx.doi.org/10.1016/S0266-3538(01)00014-8

[24] Li, J.Y. and Dunn, M.L. (2001) Viscoelectroelastic Behavior of Heterogeneous Piezoelectric Solids. Journal of Applied Physics, 89, 2893-2903. http://dx.doi.org/10.1063/1.1337595

[25] Lin, C.-H. and Muliana, A. (2013) Micromechanics Models for the Effective Nonlinear Electro-Mechanical Responses of Piezoelectric Composites. Acta Mechanica, 224, 1471-1492. http://dx.doi.org/10.1007/s00707-013-0823-4

[26] Lin, C.-H. and Muliana, A. (2014) Micromechanical Models for the Effective Time-Dependent and Nonlinear Electromechanical Responses of Piezoelectric Composites. Journal of Intelligent Material Systems and Structures, 25, 1306-1322. http://dx.doi.org/10.1177/1045389X13504477

[27] Muliana, A. (2010) A Micromechanical Formulation for Piezoelectric Fiber Composites with Nonlinear and Viscoelastic Constituents. Acta Materialia, 58, 3332-3344. http://dx.doi.org/10.1016/j.actamat.2010.02.007

[28] Jayendiran, R. and Arockiarajan, A. (2012) Modeling of Dielectric and Piezoelectric Response of 1-3 Type Piezocompo. Journal of Applied Physics, 112, 044107. http://dx.doi.org/10.1063/1.4748057

[29] Jayendiran, R. and Arockiarajan, A. (2013) Non-Linear Electromechanical Response of 1-3 Type Piezocomposites. International Journal of Solids and Structures, 50, 2259-2270. http://dx.doi.org/10.1016/j.ijsolstr.2013.03.032

[30] Aldraihem, O.J., Baz, A. and Al-Saud, T.S. (2007) Hybrid Composites with Shunted Piezoelectric Particles for Vibration Damping. Mechanics of Advanced Materials and Structures, 14, 413-426. http://dx.doi.org/10.1080/15376490701298876

[31] Aldraihem, O.J. (2011) Micromechanics Modeling of Viscoelastic Properties of Hybrid Composites with Shunted and Arbitrarily Oriented Piezoelectric Inclusions. Mechanics of Materials, 43, 740-753. http://dx.doi.org/10.1016/j.mechmat.2011.08.001

[32] Crawley, E.F. and Anderson, E.H. (1990) Detailed Models of Piezoelectric Actuation of Beams. Journal of Intelligent Material Systems and Structures, 1, 4-25. http://dx.doi.org/10.1177/1045389X9000100102

[33] Lines, M.E. and Glass, A.M. (1977) Principles and Applications of Ferroelectrics and Related Materials. Oxford University Press, Oxford.

[34] Tiersten, H.F. (1993) Electroelastic Equations for Electroded Thin Plates Subject to Large Driving Voltages. Journal of Applied Physics, 74, 3389-3393. http://dx.doi.org/10.1063/1.354565

[35] Sohrabi, A. and Muliana, A. (2013) Rate-Dependent Electro-Mechanical Coupling Response of Ferroelectric Materials: A Finite Element Formulation. Mechanics of Materials, 62, 44-59. http://dx.doi.org/10.1016/j.mechmat.2013.02.005

[36] Muliana, A. (2011) Time Dependent Behavior of Ferroelectric Materials Undergoing Changes in Their Material Prop- 
erties with Electric Field and Temperature. International Journal of Solids and Structures, 48, 2718-2731. http://dx.doi.org/10.1016/j.ijsolstr.2011.05.021

[37] Fang, D. and Li, C. (1999) Nonlinear Electric-Mechanical Behavior of a Soft PZT-51 Ferroelectric Ceramic. Journal of Materials Science, 34, 4001-4010. http://dx.doi.org/10.1023/A:1004603729657

[38] Taylor, R.L., Pister, K.S. and Goudreau, G.L. (1970) Thermomechanical Analysis of Viscoelastic Solids. International Journal for Numerical Methods in Engineering, 2, 45-59. http://dx.doi.org/10.1002/nme.1620020106

[39] King, T.R., Blackketter, D.M., Walrath, D.E. and Adams, D.F. (1992) Micromechanics Prediction of the Shear Strength of Carbon Fiber/Epoxy Matrix Composites: The Influence of the Matrix and Interface Strengths. Journal of Composite Materials, 26, 558-573. http://dx.doi.org/10.1177/002199839202600406

[40] Hussain, M., Nakahira, A., Nishijima, S. and Niihara, K. (2000) Evaluation of Mechanical Behavior of CFRC Transverse to the Fiber Direction at Room and Cryogenic Temperature. Composites Part A: Applied Science and Manufacturing, 31, 173-179.

[41] Munro, R.G. (1997) Evaluated Material Properties for a Sintered $\alpha$-Alumina. Journal of the American Ceramic Society, 80, 1919-1928. http://dx.doi.org/10.1111/j.1151-2916.1997.tb03074.x

[42] Chan, H.L.W. and Unsworth, J. (1989) Simple Model for Piezoelectric Ceramic/Polymer 1-3 Composites Used in Ultrasonic Transducer Applications. IEEE Transactions on Ultrasonics, Ferroelectrics, and Frequency Control, 36, 434-441. http://dx.doi.org/10.1109/58.31780

[43] Muliana, A. and Khan, K.A. (2008) A Time-Integration Algorithm for Thermo-Rheologically Complex Polymers. Computational Materials Science, 41, 576-588. http://dx.doi.org/10.1016/j.commatsci.2007.05.021 